ARTICLE

DOI: $10.1038 / \mathrm{s} 41467-018-04193-w$

\title{
Mutations in six nephrosis genes delineate a pathogenic pathway amenable to treatment
}

Shazia Ashraf et al."

No efficient treatment exists for nephrotic syndrome (NS), a frequent cause of chronic kidney disease. Here we show mutations in six different genes (MAGI2, TNS2, DLC1, CDK20, ITSN1, ITSN2) as causing NS in 17 families with partially treatment-sensitive NS (pTSNS). These proteins interact and we delineate their roles in Rho-like small GTPase (RLSG) activity, and demonstrate deficiency for mutants of pTSNS patients. We find that CDK20 regulates DLC1. Knockdown of MAGI2, DLC1, or CDK20 in cultured podocytes reduces migration rate. Treatment with dexamethasone abolishes RhoA activation by knockdown of DLC1 or CDK20 indicating that steroid treatment in patients with pTSNS and mutations in these genes is mediated by this RLSG module. Furthermore, we discover ITSN1 and ITSN2 as podocytic guanine nucleotide exchange factors for $\mathrm{Cdc} 42$. We generate Itsn2-L knockout mice that recapitulate the mild NS phenotype. We, thus, define a functional network of RhoA regulation, thereby revealing potential therapeutic targets. 
$\mathrm{N}$ ephrotic syndrome (NS) is the second most frequent cause of chronic kidney disease in the first three decades of life, requiring dialysis or transplantation for survival ${ }^{1}$. Based on its response to steroid treatment, steroid sensitive NS (SSNS) is distinguished from steroid resistant NS (SRNS). No efficient treatment for SRNS exists, and very little is known about disease mechanisms. Mutations in more than 40 genes have been identified as causing monogenic (single-gene) forms of NS. Interestingly, most of the encoded gene products localize to renal glomerular podocytes, confirming that podocyte loss of function is a critical part of the pathogenesis of NS and that any and all of these proteins are important for the maintenance of glomerular function $^{2,3}$. These findings have thereby helped define protein interaction complexes and functional pathways that could be targeted for future potential treatment of $\mathrm{NS}^{2,4-7}$. In addition, the mechanistic causes of steroid resistance have been a conundrum for almost six decades of their use. So far, only one causative gene (EMP2) has been discovered in $\mathrm{SSNS}^{8}$. We recently demonstrated in a large cohort of 1780 families with SRNS that in about $30 \%$ of individuals with SRNS a monogenic cause can be detected, indicating that additional single gene causes of NS must exist that are yet to be identified ${ }^{9}$.

Therefore by performing whole exome sequencing in individuals with partially treatment sensitive NS (pTSNS), we discover six novel monogenic causes for pTSNS. Strikingly, all six encoded proteins interacted within a functional module of podocytic regulation of Rho-like small GTPase (RLSG) and are at the intersection between steroid sensitivity vs. steroid resistance of NS.

\section{Results}

Mutations in six novel genes cause NS in humans. To identify additional genes mutated in NS we performed homozygosity mapping $(\mathrm{HM})^{10}$ combined with whole exome sequencing $(\mathrm{WES})^{11}$ in multiple families with NS. We also performed highthroughput exon sequencing ${ }^{12,13}$ in a worldwide cohort of $\sim 1000$ additional families with NS, examining specific candidate genes for NS based on genetic mouse models of NS.

Mice lacking Magi2 develop NS ${ }^{14-16}$. We sequenced all MAGI2 exons in 400 patients with NS. We identified homozygous truncating mutations in the MAGI2 gene (p.Gly39* and p. Tyr746 ${ }^{*}$ ) in two individuals with SRNS and neurologic impairment (Fig. 1a, b, Supplementary Table 1, Supplementary Fig. 1A). p.Gly39* was detected in an affected individual of Arab descent. p.Tyr746* was due to maternal isodisomy for chromosome 7 . We thereby discovered recessive MAGI2 mutations as a cause of SRNS with neurologic involvement in humans (Fig. 2a). Very recently, recessive mutations in MAGI2 in humans with NS have been confirmed ${ }^{17}$.

In individual A1358 of Turkish origin with early-onset NS and histology of minimal change nephrotic syndrome (MCNS) (Supplementary Table 1), HM yielded ten regions of homozygosity by descent, none of which coincided with any of the known recessive SRNS loci (Fig. 1c). By WES, we detected a novel homozygous missense mutation (p. Arg292Gln) in the TNS2 (tensin-2) gene on chromosome 12q13.13 (Supplementary Table 2). The mutation alters an amino acid residue conserved throughout evolution from $C$. intestinalis on. Direct inspection of sequence alignments did not yield a mutation in any of the 30 known SRNS genes ${ }^{3}$, and no additional homozygous truncating mutations were detected in any other gene within the mapped candidate regions. By high-throughput exon sequencing, we detected in 4 additional families (A283, 2605, A3775, and A1640) 5 additional homozygous or compound heterozygous missense mutations of TNS2 (Fig. 1d, Supplementary Table 1,
Supplementary Fig. 1B). The variant p.Ile858Met apparently represents a founder allele from India (Supplementary Table 1). Altered amino acid residues were well conserved throughout evolution (Supplementary Table 1). We thereby discovered recessive TNS2 mutations as a novel cause of NS (Fig. 2a). We introduce the term "NPHS19" for this distinct entity of NS.

Because TNS2 protein is known to interact with DLC1 (deleted in liver cancer 1$)^{18,19}$, we hypothesized that mutations of DLC1 may also cause NS. By high-throughput exon sequencing, we identified recessive mutations of DLC1 in 4 families with NS (Supplementary Table 1). Specifically, in individual A548-21 with FSGS, we detected two compound heterozygous mutations: $\mathrm{p}$. Trp $10^{\star}$ and p.Ser43Thr. In an individual of Arab descent (A496721) with SRNS and a biopsy showing focal segmental glomerulosclerosis (FSGS) (Fig. 1e), we identified two compound heterozygous mutations, p.Glu180Ala and p.Lys1358Thr, in DLC1 (Fig. 1f, Supplementary Table 1, Supplementary Fig. 1C). In an affected individual from Asia (A3118-21), we detected a homozygous mutation (p.Ala456Val) in exon 6 of DLC1 with amino acid conservation since C.elegans (Fig. 1f, Supplementary Table 1, Supplementary Fig. 1C). In family 2706 we identified a homozygous missense mutation (p.Ala1352Val) in exon 17 of DLC1 (Fig. 1f, Supplementary Table 1). We thereby discovered recessive DLC1 mutations as a novel cause of pTSNS (Fig. 2a). We introduce the term "NPHS20" for this distinct entity of NS. Interestingly, a distinct disease phenotype of partially treatment sensitive nephrotic syndrome (pTSNS) occurred in four of the five individuals with recessive TNS2 and in two of the four individuals with DLC1 mutations. pTSNS that mostly manifested between 1 and 7 years of age, with the exception of A548-21 and 2706 where the onset of NS was in adulthood (Supplementary Table 1). Interestingly, these individuals showed complete or partial response to prednisolone or cyclosporine A.

In family A5013 of Arab origin, an individual had pTSNS with the unusual renal histology of membranoproliferative glomerulonephritis (MPGN) (Fig. 1g). HM yielded 12 regions of homozygosity by descent, none of which coincided with any of the known recessive NS loci (Fig. 1h). By WES, we detected a novel homozygous missense mutation (p.Phe204Leu) in exon 6 of the CDK20 gene on chromosome 9q22.1, encoding "cyclindependent kinase 20" (Fig. 1i, Supplementary Table 1, Supplementary Table 2). The mutation alters an amino acid residue conserved throughout evolution from C. elegans on (Supplementary Fig. 1D).

By HM and WES in two siblings of Arab family A3706 (Fig. 1j, Supplementary Table 1, Supplementary Table 2) with early-onset NS we identified a homozygous missense mutation of ITSN1 (Intersectin 1) at a highly conserved amino acid residue (p. Pro180Ser). By high-throughput sequencing, we identified two additional families (A977 and A2274) with four additional compound heterozygous missense mutations of ITSN1 (Fig. 1k, Supplementary Table 1, Supplementary Fig. 1E). Although the mice lacking Itsn 1 have been shown to exhibit a neuronal phenotype ${ }^{20}$, none of individuals with early-onset NS identified here showed any brain abnormalities. We introduce the term "NPHS21" for this distinct entity of NS.

WES was also performed in five members of a Japanese family (two patients and their unaffected members) (Supplementary Fig. 2). Both affecteds in this family (Pat1, Pat 2) had pTSNS and renal histology in both the siblings showed MCNS (Fig. 11). By WES, we identified two compound heterozygous mutations in trans in ITSN2 (intersectin 2), a missense mutation (p.Ser339Cys) in exon 11 and a frame-shift deletion (p.Ile1214Serfs $\left.{ }^{\star} 2\right)$ in exon 30 of ITSN2 (Fig. 1m, Supplementary Fig. 1F, Supplementary Table 1, Supplementary Table 3). Additionally, in an individual from Arab with pTSNS (A3384), we detected a 
homozygous missense mutation (p.Glu438Gly) in exon 12 of ITSN2 (Fig. 1m, Supplementary Table 1). We thereby discovered recessive ITSN2 mutations as novel cause of pTSNS (Fig. 2a). We introduce the term "NPHS22" for this distinct entity of NS. Interestingly, all three patients (from two families) with ITSN2 mutations had pTSNS, as well as two out of four patients (from three families) with ITSN1 mutations. Similar to patients with TNS2, DLC1 or CDK20 mutations, most patients with ITSN2 or ITSN1 mutations also showed partial or complete response to Cyclosporine A.

For all mutations in all six genes segregation status was compatible with recessive inheritance (Supplementary Table 1). Supplementary Table 4 lists all the variant frequencies for all the identified mutations in the Exome Aggregation Consortium $(\mathrm{ExaC})$ and the genome aggregation database (GnomAD) along with their prediction scores using Mutation Taster (http://www. mutationtaster.org/) and Polyphen 2 (http://genetics.bwh. harvard.edu/pph2/) in silico tools. As a negative control, we performed screening for all variants that we detected in the pTSNS patients in an in-house cohort of exome data from 248 NPHP cases. This control did not reveal any homozygous carriers (Supplementary Table 4).

Three of the six proteins interact with DLC1. We discovered that four proteins MAGI2, TNS2, DLC1, and CDK20, in which we detected recessive defects as novel causes of pTSNS, interact physically or functionally to regulate RhoA/Racl/Cdc42. Specifically, we detected or confirmed the following protein-protein interactions:

First, because MAGI2 was known to interact with $\mathrm{PTEN}^{21}$, and because TNS2 contains a PTEN domain (Figs 1d and 2a), we hypothesized that MAGI2 may also interact with TNS2. By cooverexpression and co-immunoprecipitation (coIP) of wildtype and mutant cDNA clones in HEK293T cells, we found that a

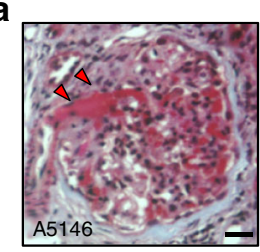

b

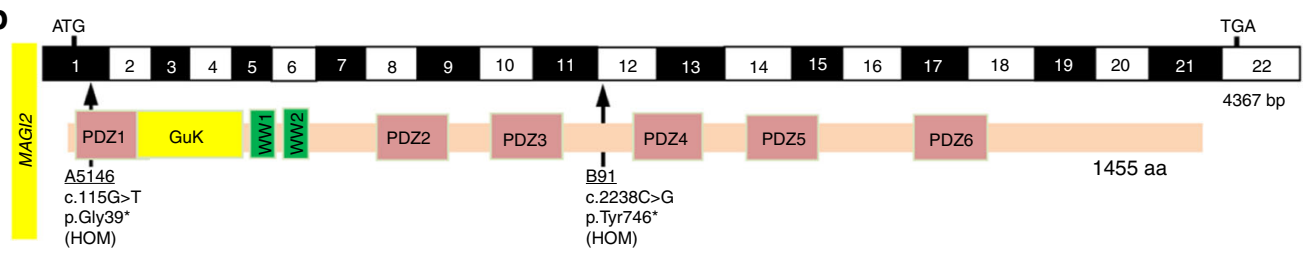

C

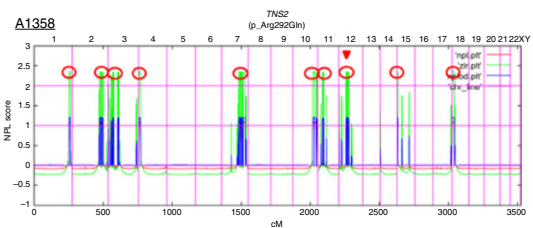

d
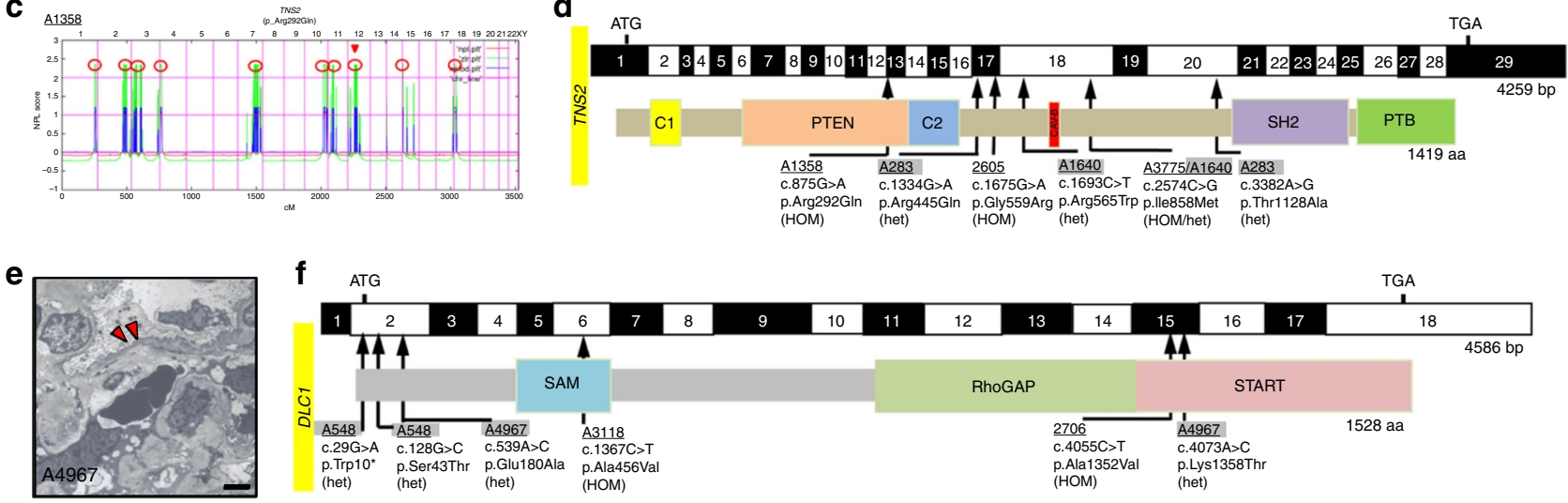

10

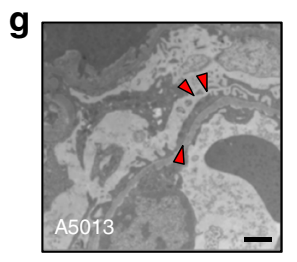

h

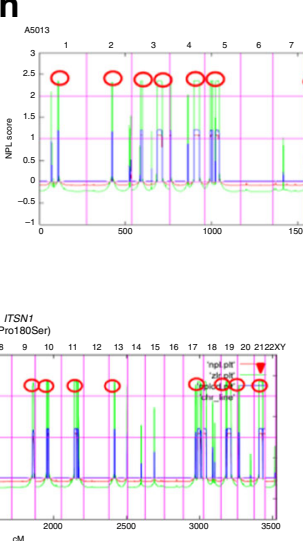

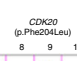

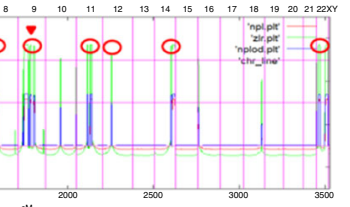

i

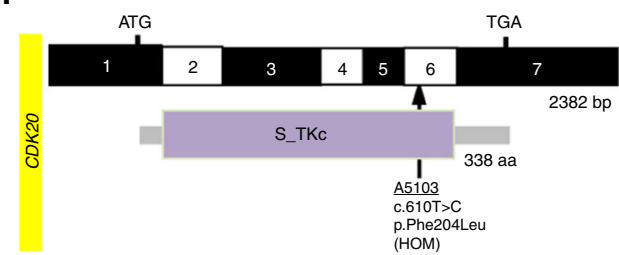

j

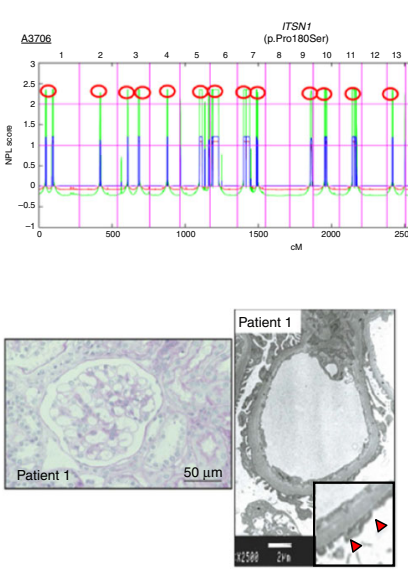

k

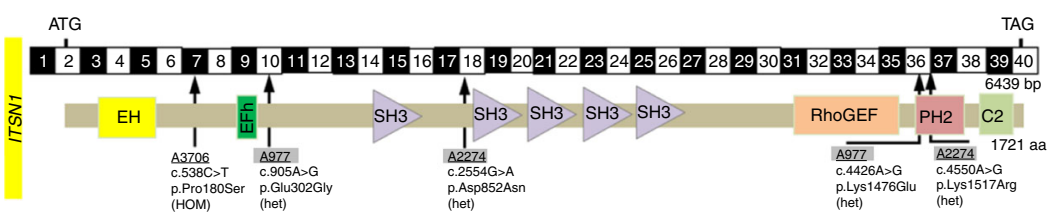

$\mathbf{m}$

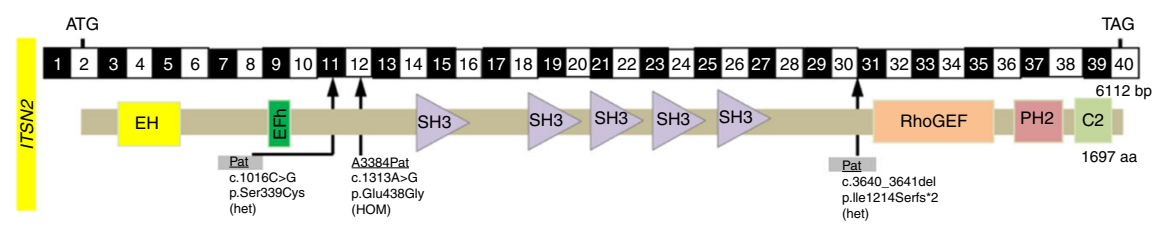


MAGI2 in fact interacts with TNS2 (Fig. 2b), which we confirmed in a confirmatory coIP (Supplementary Fig. 3A). Both mutant MAGI2 constructs, Gly39* and Tyr746* that reflect alleles of NS patients, A5146 and B91, respectively, abrogate this interaction (Fig. 2a, b). The six mutations detected in TNS2 did not abrogate interaction with MAGI2 (Supplementary Fig. 3B).

Second, MAGI2 also interacted with DLC1 (Fig. 2a, c). One mutant MAGI2 construct Gly39* reflecting the mutation of NS patient A5146 abrogates this interaction (Fig. 2c), as confirmed in a confirmatory coIP (Supplementary Fig. 4).

Third, because TNS2 had been shown to interact with DLC1 ${ }^{19}$, we performed coIP upon co-overexpression in HEK293T cells. We confirmed TNS2-DLC1 interaction (Fig. 2a), however, none of the TNS2 or DLC1 missense mutations identified in NS patients abrogated this interaction (Supplementary Fig. 5A, B).

Fourth, because we identified a CDK20 mutation in patient A5013 with pTSNS, and because a related serine/threonine protein kinase, CDK5, was shown to interact with $\mathrm{DLC1}^{22}$, we tested whether CDK20 would likewise interact with DLC1. By coIP we show that in fact CDK20 interacts with DLC1 (Fig. 2a,d) which we confirmed in a coIP (Supplementary Fig. 6A). We found that the clone reflecting DLC1 mutation Trp10* of individual A548 abrogated this interaction (Supplementary Fig. 6B). This interaction was confirmed by half endogenous Co-IPs in HEK293T cells (Supplementary Fig. 7A, B).

Finally, it was recently shown that the RhoGAP DLC1 interacts with caveolin-1 (CAV1) via DLC1's START domain ${ }^{23}$ (Fig. 2a), and that $C A V 1$ expression is regulated by $E M P 2^{24}$ (Fig. 2a). This regulation was shown to be steroid sensitive ${ }^{25}$. Furthermore, we had shown that EMP2 mutations were the only monogenic cause of SSNS known so far in humans ${ }^{8}$ as well as in a zebrafish model of $\mathrm{SSNS}^{25}$. To determine whether CAV1 could directly be involved in DLC1 regulation, we performed coIP between DLC1 and CAV1 by co-overexpression in HEK293T cell. We confirmed that DLC1 interacts with CAV1 (Fig. 2a, e), and showed that the mutant Lys1358Thr, reflecting the allele of NS patient A4967 and present in CAV-1 binding site in $\mathrm{DLC1}^{23}$, abrogated this interaction (Fig. 2e). To further test the effect of steroids on this interaction, we performed reciprocal coIP between cooverexpressed DLC1 and CAV1 in HEK293T cells that were pre- and post-treated with $100 \mathrm{uM}$ of dexamethasone (Supplementary Fig. 8). However, this treatment did not alter the results shown in Fig. 2e. We demonstrated by co-overexpression in
HEK293T cells that CAV1 also interacts with TNS2 and MAGI2 (Supplementary Fig. 9A, B).

In summary, we identified in patients with pTSNS recessive mutations in six different genes, the products of which mutually interact within a complex that contains the RhoGAP DLC1. The finding that specific mutations abrogated some of these interactions most likely reflects allele-specific pathogenic effects of these mutations.

Furthermore, we also investigated the endogenous expression of TNS2 and DLC1 by immunofluorescence microscopy (IF) in human podocytes and found that both TNS2 and DLC1 colocalize with an antibody labeling phosphotyrosine at focal and fibrillar adhesions, consistent with the role of focal adhesions in podocyte migration ${ }^{26}$ and the pathogenesis of $\mathrm{NS}^{27,28}$ (Supplementary Fig. 10). Additionally, by IF we show that upon overexpression, full length GFP-MAGI2 colocalizes with $\beta$ catenin at adherens junctions. In contrast, overexpression of GFP-MAGI2 Gly39* and Tyr746*, representing mutations in pTSNS, fail to localize at adherens junctions (Supplementary Fig. 11).

Circular dichroism modeling of mutation in SAM domain of DLC1. Since one of the mutations (p.Ala456Val) in DLC1 is located in its SAM domain, we studied effect of this mutation on its secondary structure by performing circular dichroism (CD) assay. The wild type (WT) and mutant (Ala19Val in SAM domain) proteins did not show any difference in their CD spectrum indicating overall similar three dimensional fold (Supplementary Fig. 12A). We then recorded the melting spectrum of WT and mutant proteins and found that the denaturation temperature of WT SAM domain was near to $58^{\circ} \mathrm{C}$ and the melting curve was sigmoidal indicating cooperative unfolding behavior. However, for Ala19Val SAM, there was loss of cooperativity and absence of sharp melting point (Supplementary Fig. 12B). Arg823Trp mutation in SAM domain of ANKS6 has been previously reported to cause structure destabilization and responsible for polycystic kidney disease ${ }^{29}$. Interestingly, both Arg823Trp (Arg64) mutation in ANKS6 and Ala456Val (Ala19) mutation in DLC1 are located in proximity in DLC1-SAM domain structure indicating a possible link of similar destabilization effect (Supplementary Fig. 12C, D).

\footnotetext{
Fig. 1 High-throughput sequencing reveals recessive mutations of MAGI2, TNS2, DLC1, CDK20, ITSN1, or ITSN2 as causing NS in humans. a Renal histology of individual A5146-21 with focal segmental glomerulosclerosis (FSGS) and MAGI2 mutation (scale bar $=50 \mu \mathrm{m}$ ). $\mathbf{b}$ Exon structure of human MAGI2 cDNA and mutations. Below is the protein domain structure of MAGI2, showing GuK, WW1, WW2, and six different PDZ domains. Two different homozygous truncating mutations of MAGI2 were detected in two families with NS and neurological impairment. c Homozygosity mapping identifies ten recessive candidate loci (red circles) in family A1358 with NS, and WES identifies a homozygous mutation of TNS2 (p.Arg292GIn). Non-parametric lod scores (NPL) were calculated and plotted across the human genome. The TNS2 locus (arrowhead) is positioned within one of the maximum NPL peaks on chromosome 12q. d Exon and protein domain structure of human TNS2. Six different TNS2 mutations were detected in five families with NS. Family numbers and amino acid changes are given (Supplementary Table 1). Arrow heads denote altered nucleotides. Lines and arrows indicate positions of mutations in relation to exons and protein domains. Family numbers with compound heterozygous mutations (het) are highlighted in gray. e Renal histology of individual A4967-21 with FSGS and DLC1 mutations. TEM reveals podocyte foot process effacement (arrow heads, magnification $8000 \mathrm{x}$ ). $\mathbf{f}$ Exon and protein domain structure of human DLC1. The SAM, RhoGAP, and START domains are depicted by colored bars, in relation to encoding exon position. Six different DLC1 mutations were detected in four families with NS. Positions of amino acid changes (Supplementary Table 1) are marked with arrowheads. $\mathbf{g}$ Renal histology of individual A5013-21 with membranoproliferative glomerulonephritis (MPGN) and mutation in CDK20. TEM reveals podocyte foot process effacement and thickening of glomerular basement membrane (arrow heads, magnification 7000x). h Homozygosity mapping identifies twelve recessive candidate loci (red circles) in family A5013 with NS, and WES identifies a homozygous mutation of CDK20 (p.Phe204Leu), positioned within one of the maximum NPL peaks on chromosome 9q. i Exon and protein domain structure of human CDK20. The serine threonine kinase (S_TKc) domain is depicted by a colored bar, in relation to encoding exon position. One homozygous mutation in CDK20 was detected in family A5013 with NS. $\mathbf{j}$ Homozygosity mapping in family A3706 with NS identifies 17 recessive candidate loci (red circles), and WES identifies a homozygous mutation of ITSN1 (p.Pro180Ser), positioned within one of the maximum NPL peaks on chromosome 21q. $\mathbf{k}$ Exon and protein domain structure of human ITSN1. Five different ITSN1 mutations were detected in three families with NS. I Renal histology of Patient-1 with mutations in ITSN2 revealed minimal change disease (scale bar $=50 \mu \mathrm{m}$ ). EM showed foot process effacement (scale bar $=2 \mu \mathrm{m})$. $\mathbf{m}$ Exon and protein domain structure of human ITSN2. Three different ITSN2 mutations were detected in two families with NS
} 
Knockdown of MAGI2 or CDK20 or DLC1 causes a decrease in cell migration rate. To elucidate the role of the genes of this RLSG regulatory module in the pathogenesis of pTSNS, we next studied cell migration rate, which is typically altered upon loss of function of NS-causing genes. We show that knockdown of
MAGI2 or CDK20 in cultured human podocytes results in a reduced migratory phenotype (Supplementary Fig. 13A, B). Similarly, knockdown of DLC1 in both IMCD3 cells and human podocytes also show a reduction in cell migration (Fig. $2 \mathrm{f}$, Supplementary Fig. 14). This effect is found to be reversed

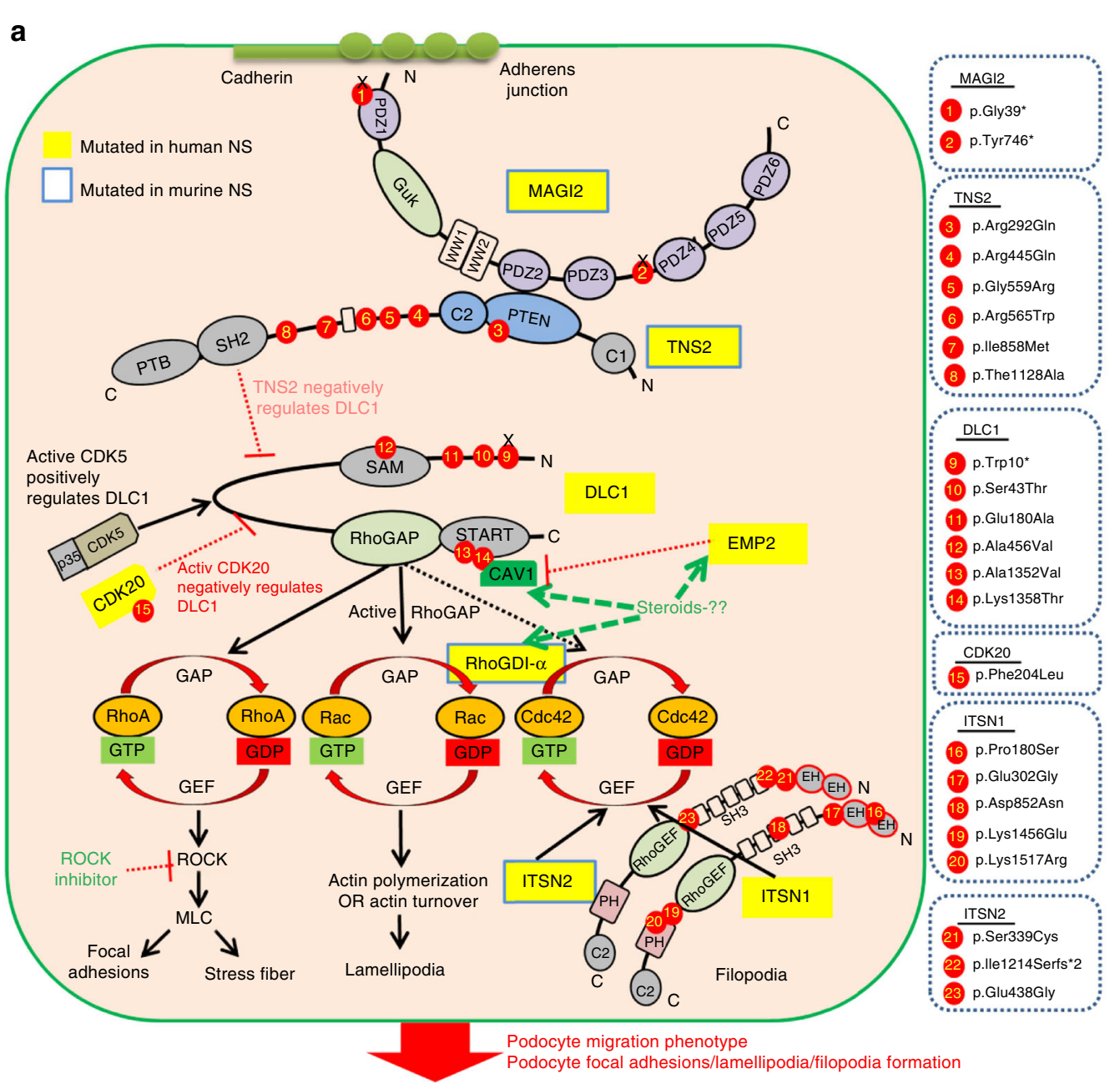

b

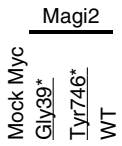

GFP Magi2 + + + +

Myc TNS2 WT -+++ Blot

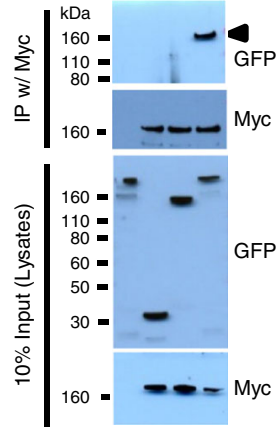

C

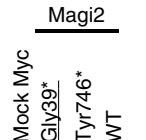

GFP Magi ++++

Myc DLC1 WT - + + + Blot

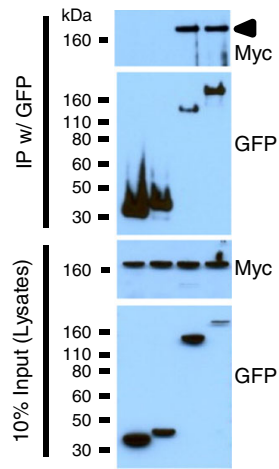

d

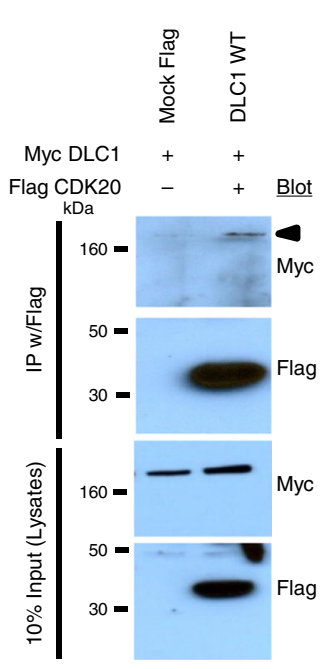

e

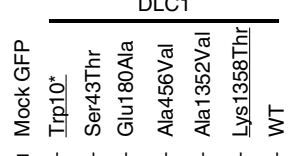

$\mathbf{f}$

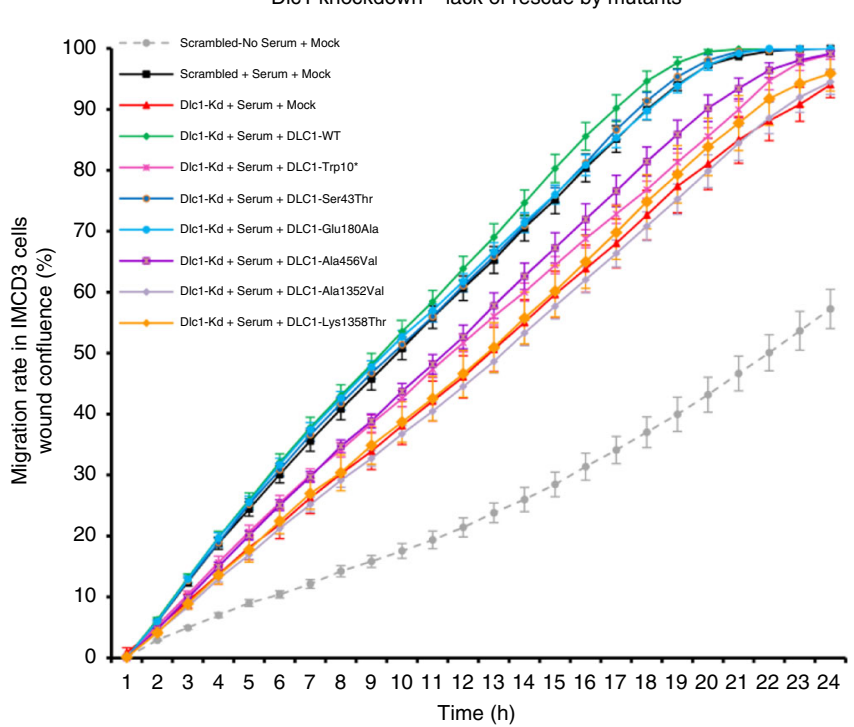


when WT human DLC1 is transfected to IMCD3 cells (Fig. 2f). In contrast, four out of six DLC1 mutants (Trp10*, Ala456Val, Ala1352Val, and Lys1358Thr), detected in pTSNS, failed to fully rescue this decreased migratory phenotype. The knockdown of TNS2 did not show any effect on podocyte migration (Supplementary Fig. 15).

RhoA regulation by this NS pathogenic module. Because defects in the regulation of Rho-like small GTPases have been implicated in the pathogenesis of monogenic SRNS ${ }^{4,5,30}$, we evaluated the effect of overexpression and transient knockdown of all six members of NS pathogenic module on the regulation of RhoA/ Rac1/Cdc42 activity (Supplementary Fig. 16, Supplementary Table 5) and its potential impairment by the mutations detected in patients with pTSNS. We further evaluated the effect of stable knockdown of TNS2 and DLC1 in human podocytes, on the regulation of $\mathrm{RhoA} / \mathrm{Racl} / \mathrm{Cdc} 42$ activity. Our results were consistent with the findings observed with the transient knockdown for these two genes (Supplementary Fig. 17).

No effect of overexpression or knockdown of TNS2, CDK20, ITSN1, or ITSN2 was found on the regulation of active (GTPbound) Rac1, with the exception of an increase of active Rac1 upon knockdown of DLC1 (Supplementary Figs 16 and 17, Supplementary Table 5). This finding most likely indicates an effect of the known crosstalk between RhoA and Rac1 signaling.

We found that overexpression of MAGI2 increased active (GTP-bound) RhoA, whereas overexpression of the MAGI2 mutants detected in patients with pTSNS lacked this effect (Fig. 3a, Supplementary Table 5). We tested the effect of TNS2 on RhoA regulation and found that TNS2 behaved similar to MAGI2 (Fig. 3b). Because we had shown that CDK20 interacts with the RhoGAP DLC1 (Fig. 2a, d), we tested the effect of CDK20 on RhoA regulation and found that CDK20 behaved similar to MAGI2 and TNS2 in that overexpression of CDK20 increased active RhoA, whereas overexpression of the CDK20 mutant detected in patients with pTSNS lacked this effect (Fig. 3c). We then tested the effect on RhoA regulation of the RhoGAP DLC1, which interacts with MAGI2, TNS2, and CDK20. We found that DLC1 behaved opposite to MAGI2, TNS2, and CDK20 because overexpression of DLC1 decreased active RhoA. Overexpression of the DLC1 mutants detected in patients with pTSNS again lacked this effect (Fig. 3d). Reciprocally, knockdown of MAGI2, TNS2, and CDK20 decreased active RhoA, which was rescued by overexpression of respective WT cDNAs, whereas all or most of the mutants detected in patients with pTSNS lacked this effect
(Fig. 3e-g, Supplementary Table 5). However, knockdown of DLC1 was found to increase active RhoA (Fig. $3 \mathrm{~h}$ ). We thereby clearly showed that the mutations detected in individuals with pTSNS in the six genes of this newly discovered RhoA regulatory module are an important part of the pathogenesis of pTSNS. We also tested the effect of $C A V 1$ on RhoA activation and found that its overexpression decreases active RhoA, whereas its knockdown increased active RhoA (Supplementary Fig. 18).

We then tested the effect of double-knockdown of TNS2 and DLC1 on RhoA activity and found that the decrease in active RhoA by TNS2 knockdown or increase in active RhoA by $D L C 1$ knockdown was normalized (Fig. 3i, Supplementary Table 5). To test if the TNS2 expression modulates the RhoA activity via DLC1, we knockdown DLC1, followed by overexpression of WTTNS2 in HEK293T cells and found the increase was comparable to knockdown of DLC1 alone (Fig. 3j). This confirms that the TNS2 is upstream of DLC1 and works via DLC1 on RhoA regulation. Similarly, knockdown of DLC1 and CDK20 together (Fig. 3k) also showed that both CDK20 and MAGI2 affect RhoA regulation via DLC1, which plays a central role in this pathogenesis. In contrast, the double-knockdown of DLC1 and MAGI2 did not (Fig. 31).

Defects of RhoA regulation are mitigated by steroids. Since most of the individuals found here with mutations in MAGI2, TNS2, DLC1, or CDK20 genes had clinically shown benefits by treatment with steroids, we next tested if steroid treatment with dexamethasone in different concentrations will show any the effect on the increase of RhoA activity upon DLC1 knockdown in HEK293T cells. Wild type HEK293T cells showed no effect of dexamethasone treatment on active RhoA, while they responded to RhoA activators (red) and RhoA inhibitors (blue) as a positive controls (Supplementary Fig. 19). In contrast, treatment with dexamethasone at $>75 \mu \mathrm{M}$ abolished the effects on RhoA activation by knockdown of $D L C 1$ (Fig. $3 \mathrm{~m}$ ). We observed similar treatment effect of dexamethasone $(>75 \mu \mathrm{M})$ on RhoA activation via knockdown of $C D K 20$ (Fig. 3n). However, no treatment effect of dexamethasone $(<100 \mu \mathrm{M})$ was seen on RhoA activation via knockdown of MAGI2, TNS2, or CAV1 (Fig. 3n). We further confirmed these findings in human podocyte cells (Supplementary Fig. 20). This indicates that the beneficial effect of steroids in pTSNS and mutations in these genes is likely mediated by the RhoA-regulating module.

Discovery of ITSN1 and ITSN2 as two NS-relevant GEFs for Cdc42. We here discover recessive mutations in ITSN1 and

Fig. 2 Gene products of MAG12, TNS2, DLC1, CDK20, and CAV1 physically and functionally interact to regulate RhoA/Rac1/Cdc42 activation. a Identification of six novel monogenic causes of NS reveals a regulatory network of RhoA activation. The large rounded square symbolizes a podocyte. All six proteins MAGI2, TNS2, DLC1, CDK20, ITSN1, and ITSN2, in which recessive defects were detected herein as novel causes of pTSNS, interact physically or functionally to regulate RhoA/Rac1/Cdc42. Yellow labels highlight proteins encoded by genes, which if mutated give rise to monogenic nephrosis as shown in this study (MAGI2, TNS2, DLC1, CDK20, ITSN1, and ITSN2) or as published (EMP2, ARHGDIA). Blue frame around yellow labels indicate that there is also a monogenic mouse model of NS or a zebrafish model known, as shown in this study for ITSN2 or as published for MAGI2, TNS2, EMP2, and RhoGDI$\alpha$. For each of the proteins, MAGI2, TNS2, DLC1, CDK20, TLN1, or ITSN1, the protein domains are shown. Red circles denote positions of mutations that we found in patients. Truncation mutations are represented by " $x$ ". By identifying novel monogenic causes of NS we discovered a cluster proteins that regulate Rho/Rac/Cdc42 activation as being central for the pathogenesis of these patients. b MAGI2 interacts with TNS2 upon co-overexpression and coimmunoprecipitation (colP) in HEK293T cells. Both mutant MAGI2 clones, Gly39* and Tyr746* (underlined) that reflect alleles of NS patients A5146-21 and B91 respectively, abrogate this interaction. c MAGI2 interacts with DLC1 upon co-overexpression and colP in HEK293T cells. One mutant MAGI2 clone Gly39* (underlined) reflecting a mutation of NS patient A5146-21 abrogates this interaction. d DLC1 interacts with CDK20 upon co-overexpression in HEK293T cells. e DLC1 interacts with CAV1. Two mutant DLC1 c-DNA clones, reflecting Trp10* and Lys1358Thr alleles of NS patients A548-21 and A496721 respectively, lack this interaction. $\mathbf{f}$ In IMCD3 cells, migration rate is induced in the presence of serum as compared to scrambled control. Knockdown of DIc1 in IMCD3 cells using mouse DIc1 siRNA \#1 impairs cell migration rate (red vs. black curve with serum). The decrease in migration is rescued by transfection with full-length human DLC1 cDNA (green curve). Transfection with four out ot six mutants (Trp10*, Ala456Val, Ala1352Val, and Lys1358Thr) failed to rescue this migratory phenotype 
ITSN2 as causing NS. Both proteins are members of the guanine exchange factor (GEF) family of proteins that activate Cdc $42^{31}$ (Fig. 2a). To test whether loss of function of ITSN1 and ITSN2 GEF activity causes a nephrosis phenotype in humans, we examined the effect of mutations identified in patients with
pTSNS on the active states of Cdc42. We show that overexpression of WT-ITSN1 results in a significant increase in active Cdc42 in HEK293T cells (Fig. 4a) or human podocytes (Supplementary Fig. 21). All of the ITSN1 mutants detected in patients with pTSNS lacked this effect, demonstrating its relevance for the
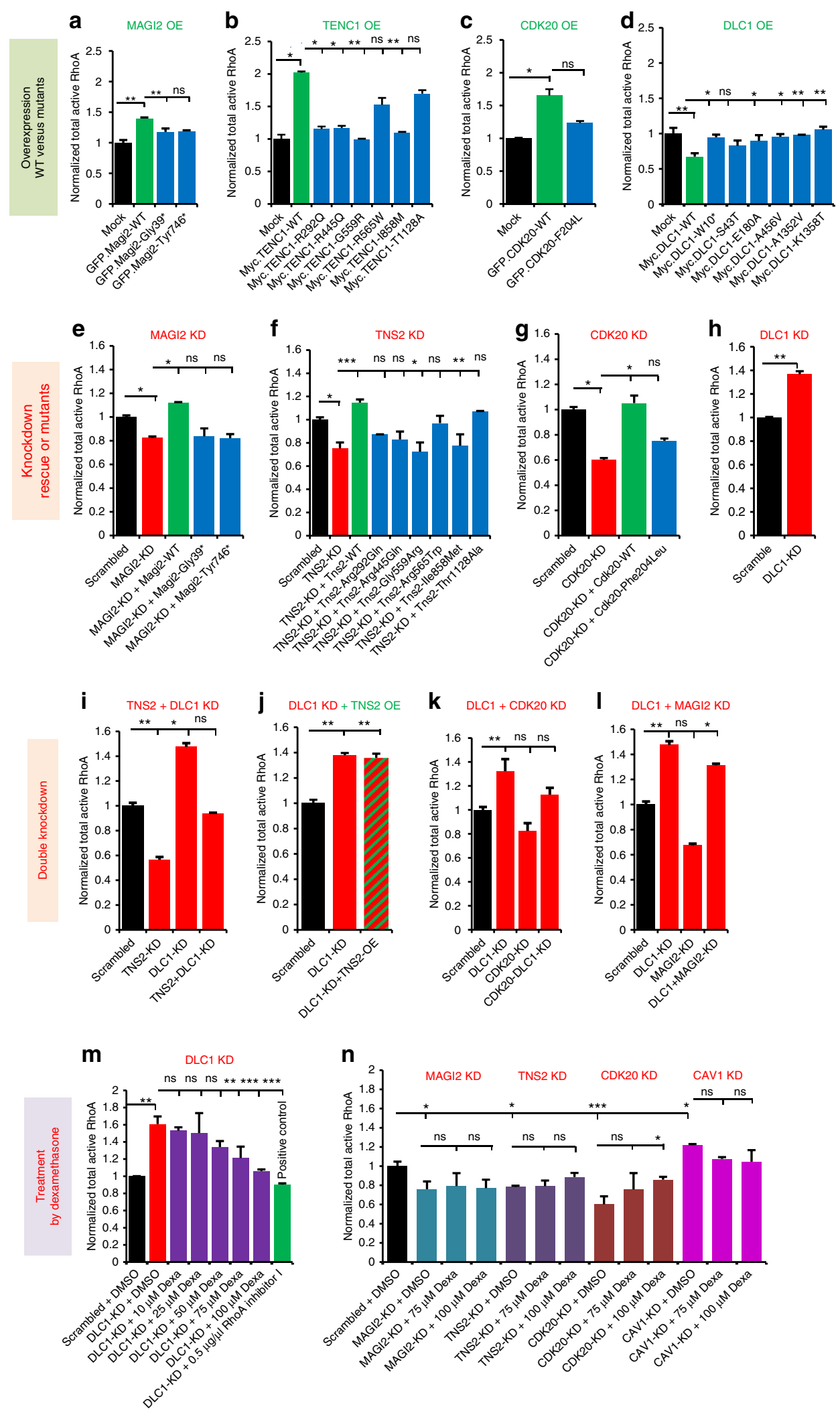
pathogenesis of NS. Similar results were obtained for ITSN2 where all the mutations detected in pTSNS patients lacked the effect of Cdc42 activation (Fig. 4b). Filopodia, thin actin-rich plasma-membrane protrusions, play an important role in cell migration and wound healing 32 and $\mathrm{Cdc} 42$ is a key protein involved in filopodia formation ${ }^{33}$. Thus, we examined the effect of ITSN1 and ITSN2 mutations on filopodia induction in cultured human podocytes. Human podocyte cells were co-transfected with Myc-Cdc42 and FLAG tagged WT-ITSN1, five mutants discovered in pTSNS patients (Pro180Ser, Glu302Gly, Asp852Asn, Lys1476Glu, and Lys1517Arg) or flag-mock and images were captured (Supplementary Fig. 22). WT-ITSN1 transfected cells induced dense filopodia all around the cell surfaces (Supplementary Fig. 22). Overexpression of ITSN1 mutants resulted in a significant decrease in the ratio of cells with filopodia compared to WT-ITSN1 (Fig. 4c). Similar loss of filopodia induction was observed for overexpression of ITSN2 wild type vs. three mutants discovered in pTSNS patients (Ser339Cys, Glu438Gly and Ile1214Serfs²) (Fig. 4d, Supplementary Fig. 23). We thus demonstrated that the ITSN1 and ITSN2 mutations detected from the pTSNS families cause loss of function in Cdc42-GEF activity and also inhibit filopodia induction in cultured human podocytes cells. The other genes were not tested because their overexpression or knockdown altered RhoA activity, which induces focal adhesions, rather than Cdc42, which induces filopodia.

Generation of Itsn 2 deficient mice. To evaluate the pathogenic roles of ITSN2 mutations in the pTSNS disease phenotype, we generated Itsn- $L \mathrm{KO}$ mice. In the $\mathrm{KO}$ mice, a region including exon 31 of Itsn2 (NC_000078) was replaced with the targeting vector throughout the entire body (Supplementary Fig. 24A). This region encodes a part of the DH domain, affecting Cdc42-GEF activity. The expression level of Itsn2-S and Itsn2-L mRNA in various organs were determined by reverse transcription PCR (RT-PCR; Supplementary Fig. 24B). Itsn2-S and Itsn2-L mRNA were expressed ubiquitously in wild type mice. In the homozygous KO (Itsn $2^{\mathrm{L}-\mathrm{l}-}$ ) mice, Itsn2-L mRNA was not detected (Supplementary Fig. 24B). Itsn2 $\mathrm{L}-/ \mathrm{L}-$ mice were normal in appearance, growth, development, and fertility. There was no difference between Itsn $2 \mathrm{~L}^{-} \mathrm{L}-$ and wild-type mice in the histologic findings of the kidneys (Fig. 4e) and in the level of urinary protein (Fig. $4 \mathrm{f}$ at $0 \mathrm{~h}$ ) during the course of natural history. These results suggested that Itsn2-L is not necessary for kidney development in mice. Patients with NS often relapse after an upper respiratory tract infection ${ }^{34}$. We hypothesized that some interventions that mimic infectious events may trigger the onset of a nephrosis phenotype in Itsn $2^{\mathrm{L}-/ \mathrm{L}-}$ mice. We used the LPS model of transient nephrotic syndrome, in which foot process effacement and massive proteinuria of wild-type mice develop within $12-24 \mathrm{~h}$ and return to baseline levels within $72 \mathrm{~h}^{35}$. The time course of albuminuria was measured in wild type, Itsn $2^{+/ L}-$ and Itsn $2 \mathrm{~L}-/ \mathrm{L}-$ mice at various time points before and after the injection of LPS (Fig. 4f). There was no difference in urine albumin between wild-type and $\operatorname{Isn} 2^{\mathrm{L}-/ \mathrm{L}-}$ mice before the injection of LPS. Urine albumin was increased in $I_{s n} 2^{\mathrm{L}-/ \mathrm{L}-}$ mice from 12 to $48 \mathrm{~h}$ after LPS injection, with statistically significant differences at 12 and $36 \mathrm{~h}$ compared with WT mice and Its $n 2^{+/ \mathrm{L}-}$ mice. At $36 \mathrm{~h}$ of observation, albuminuria continued to be elevated in Itsn $2^{\mathrm{L}-/ \mathrm{L}-}$ mice, while it started to decline in WT and Itsn $2^{+/ L}-$ mice. In all groups, urine albumin returned to baseline at $60 \mathrm{~h}$. To confirm whether the urinary phenotype reflected pathologic findings, we examined the electron microscopy of mice kidneys. LPS injection induced foot process (FP) effacement in both WT and Itsn $2^{\mathrm{L}-/ \mathrm{L}-}$ mice within $24 \mathrm{~h}$. At $48 \mathrm{~h}$ after LPS injection, FP were almost recovered in WT mice, while diffuse FP effacement was observed in Itsn $2^{\mathrm{L}-/ \mathrm{L}-}$ mice (Fig. $4 \mathrm{~g}$ ). We thereby demonstrated that ITSN2-L knockout caused delayed recovery from podocyte injury. This finding adds yet another example of a pathogenic role of small Rho-like GTPases (here Cdc42), in which there is incomplete penetrance of the disease phenotype, analogous to the partially treatment sensitive phenotype (pTSNS) seen in mutations in the RhoA regulatory module members-MAGI2, TNS2, DLC1, and CDK20 (Fig. 2a).

\section{Discussion}

We here discover recessive mutations of MAGI2, TNS2, DLC1, CDK20, ITSN1, and ITSN2 genes as novel causes of NS. Whereas over 50 monogenic genes have been published to cause steroid resistant nephrotic syndrome (SRNS), if mutated, there is virtually no monogenic cause known for steroid sensitive nephrotic (SSNS) syndrome, the latter including steroid dependent (SDNS) and frequently relapsing nephrotic syndrome (FRNS). Thus, SRNS and SSNS have always appeared as molecularly and functionally completely separate entities. Therefore our finding that nine of the 12 patients with mutations in one of the six genes of

Fig. 3 The novel RhoA regulatory network of MAGI2, TNS2, DLC1, CDK20, and CAV1 is a central part of the pTSNS pathogenesis. a Overexpression of GFP-tagged MAGI2 wild type (WT) but not the two MAGI2 mutants from pTSNS patients resulted in a significant increase in active RhoA. Active RhoA was measured by G-LISA assay in HEK293T cells. b Myc-tagged TNS2-WT increased active RhoA levels upon overexpression in HEK293T cells as compared to Mock. However, all six TNS2 mutant clones from pTSNS patients failed to increase active RhoA. c GFP-tagged CDK20-WT showed a significant increase in active RhoA level upon overexpression in HEK293T cells, while the mutant clone Phe240Leu, from a pTSNS patient, failed to do so. $\mathbf{d}$ Myc-tagged DLC1-WT showed a significant decrease in active RhoA level upon overexpression in HEK293T cells as compared to Mock. However, none of the six DLC1 mutant clones from pTSNS patients showed this effect. e siRNA mediated knockdown of MAGI2 decreased active RhoA. The overexpression of Magi2-WT rescued this effect while both the mutants from pTSNS patients did not. $\mathbf{f}$ siRNA mediated knockdown of TNS2 decreased active RhoA. The overexpression of TNS2-WT rescued this effect, while four out of six TNS2 mutant clones from pTSNS patients did not. $\mathbf{g}$ siRNA mediated knockdown of CDK20 decreased active RhoA. This effect was rescued by the overexpression of CDK20-WT, but not by the overexpression of mutant clone Phe240Leu. $\mathbf{h}$ siRNA mediated knockdown of DLC1 showed a significant increase in active RhoA. i siRNA mediated knockdown of TNS2 or DLC1 in HEK293T cells decreased or increased active RhoA levels, respectively, as compared to scrambled control. The parallel knockdown of both TNS2 and DLC1 rescued this effect on active RhoA. $\mathbf{j}$ siRNA mediated knockdown of DLC1 increased active RhoA and this effect was unaltered upon overexpression of TNS2 in DLC1 knockdown cells, suggesting that TNS2 modulates RhoA activity indirectly via DLC1. k siRNA mediated knockdown of DLC1 or CDK20 in HEK293T cells increased and decreased active RhoA levels, respectively, as compared to scrambled control. The combined knockdown of both DLC1 and CDK20 rescued this effect on active RhoA. I siRNA mediated knockdown of DLC1 or MAGI2 in HEK293T cells increased and decreased active RhoA levels, respectively, as compared to scrambled control. The combined knockdown of both DLC1 and MAGI2 failed to rescue this effect on active RhoA. $\mathbf{m}$ Treatment with dexamethasone (75 and $100 \mu \mathrm{M})$ inhibited in a dose-dependent manner the increase of active RhoA elicited by DLC1 knockdown. $\mathbf{n}$ The change in active RhoA upon knockdown of CDK20, but not MAGI2, TNS2, or CAV1 was found reversible by treatment of HEK293T cells with $100 \mu$ M dexamethasone. Error bars are defined as the standard error of at least three independent experiments. One-way ANOVA with Dunnet's post hoc test was performed vs. control. Data are presented as the mean \pm SEM. ${ }^{\star} P<0.05$, ${ }^{\star \star} P<0.01,{ }^{\star \star \star} P<0.001$. ns, non significant 
the "Rho-like small GTPase regulatory cluster" had a partially treatment responsive form of nephrotic syndrome is quite striking and has not been described in a similar way before. This finding confirms the unique role of the pathway discovered that maybe amenable to therapy.

RhoA/Rac1/Cdc42 regulation has been implicated in SRNS before $^{36}$ and few genes that code the regulators of Rho GTPases were found to be associated with human glomerular disease. We showed that ARHGDIA, which encodes a GDI for Cdc42 and Rac1, causes autosomal recessive SRNS 4 . In addition, patients with NS and mutations in KANK2, which encodes kidney ankyrin repeat-containing protein 2 , exhibited increased active RhoA in podocytes $^{5}$. Furthermore, we demonstrated that mutations in FAT1 cause familial focal segmental sclerosis (FSGS) via defective Rho GTPases ${ }^{30}$. Podocyte-specific loss of Cdc42 in mice has been described to lead to foot process effacement and congenital

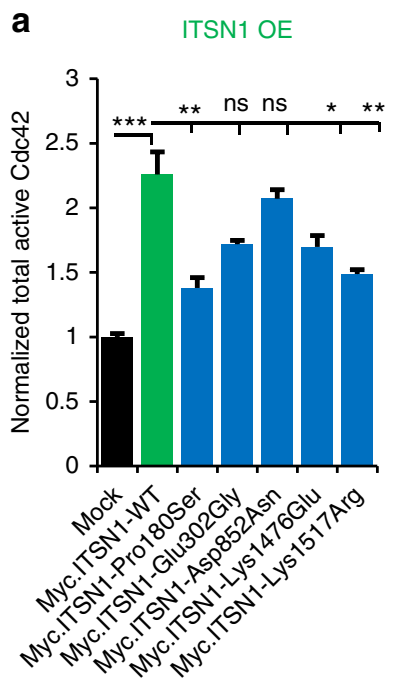

b ITSN2 OE C
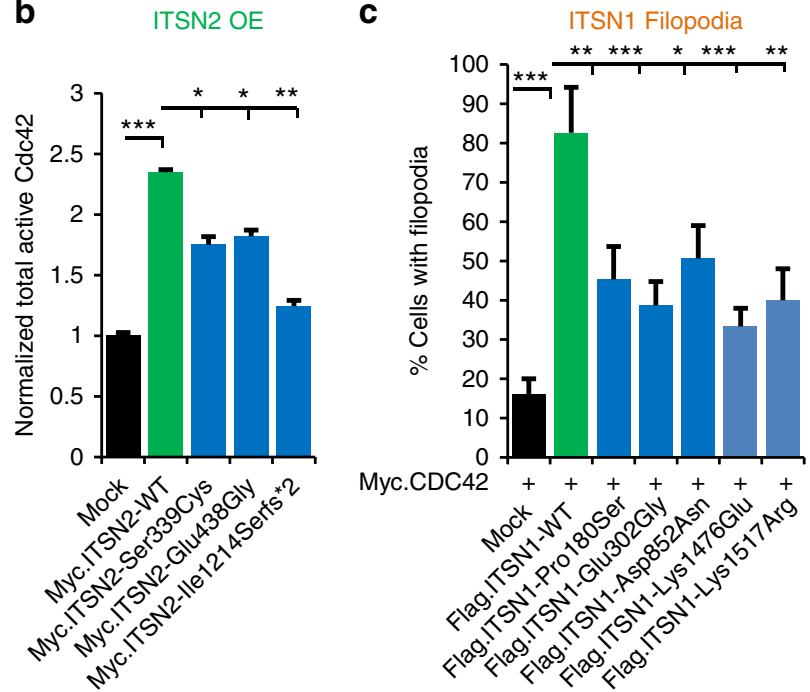

d

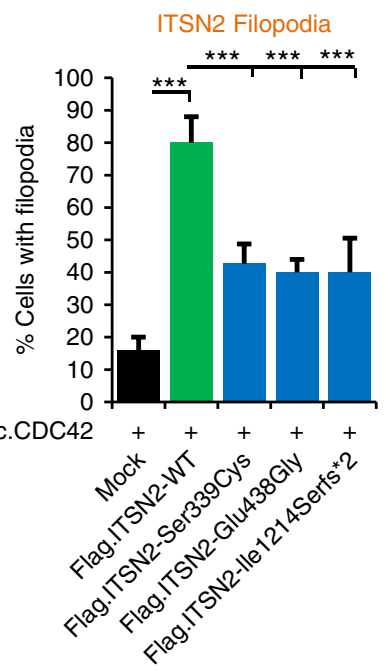

e
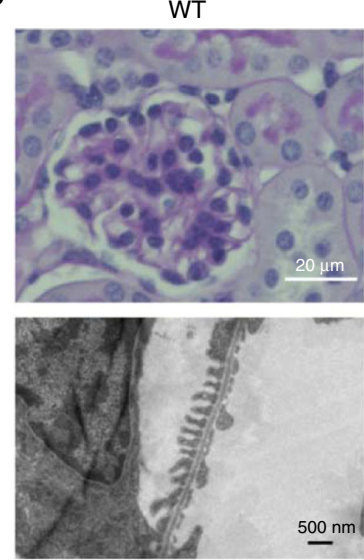

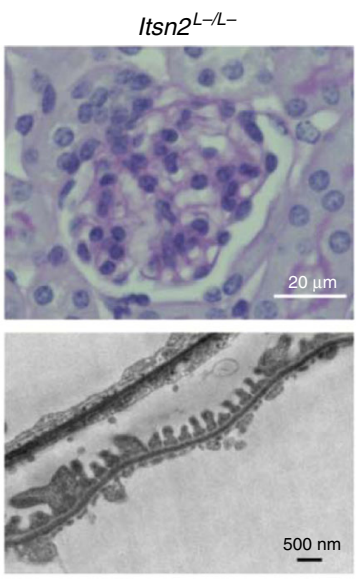

f

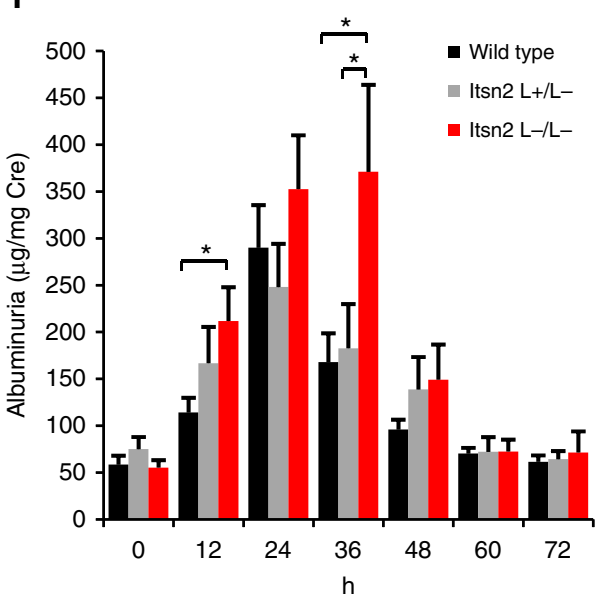

g
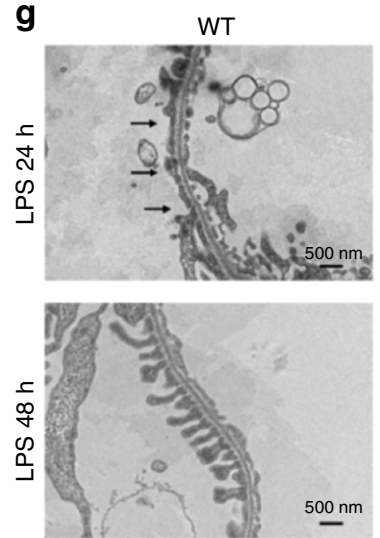
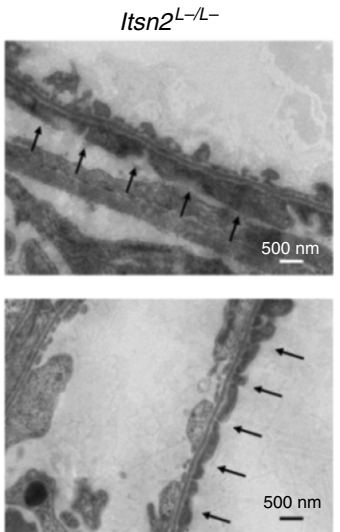
proteinuria $^{37}$. It has been suggested that there is strong crosstalk between the three Rho-like small GTPases RhoA, Rac1, and Cdc $42^{38}$. Therefore, we here evaluated activation of all three Rholike small GTPases by G-LISA for each of the six novel monogenic causes of pTSNS. In contrast to previous findings showing a role of Racl in SRNS of humans, mice and zebrafish ${ }^{4}$, and a role of Cdc42 in NS in mouse models ${ }^{37}$, we here discovered that for four different novel genes (MAGI2, TNS2, CDK20, and DLC1), which if mutated cause pTSNS, modulation of activated RhoA rather than Racl or Cdc42 is central to the pathogenesis (Supplementary Table 6). This may indicate that in pTSNS caused by mutations in these four genes impairment of the RhoA-driven function of focal adhesions is more relevant for the pathogenesis than impairment of Rac1-driven lamellipodia formation.

Mechanisms of steroid action in NS have been a conundrum for the six decades of their use. Interestingly, we found that the effects of KD of DLC1 or CDK20 on RhoA activation in human podocytes and HEK293T cells were abolished by steroid treatment with dexamethasone. We here generate evidence of steroid action on podocyte function indicating that this newly delineated RLSG regulatory module mediates the beneficial effect of steroids in patients with pTSNS and mutations in these genes. We cannot evidently answer why steroids help cure NS in some patients, and it has been only known that steroids may influence the transcription of a large number of genes by virtue of their interaction with intracellular receptors. However, our data suggests that steroids might target a specific protein or modulator of protein, which is most likely a part of the complex described in this manuscript, rather than the transcriptional target through the receptors. Further studies of this protein interaction module may reveal the specific target(s) of steroid action in podocytes.

Tns2-deficient mice have been previously described to develop mild glomerular injury on a DBA/2 background, but not on a C57BL/6J or a 129/SvJ background, suggesting that glomerular injury by the deletion of Tns 2 was modified by the genetic background ${ }^{39}$. In mice, LPS stimulates toll-like receptor-4 (TLR4) and upregulates CD80 expression on podocytes, resulting in foot process effacement and transient proteinuria ${ }^{35}$. Here, we generated the Itsn2-L knockout mice with partially penetrant proteinuria following LPS injection. This mouse model exhibits an NS phenotype that is at an interphase between plain steroid resistance and steroid sensitivity. We observed that the individuals with ITSN2 mutations had frequently relapsing NS while the Itsn2-L knockout mouse showed inducible and transient proteinuria. In humans, the severity of SSNS varies, where some patients show complete remission with steroid therapy while some have frequently relapsing NS. The study by Wharram et al. published in J. Am. Soc. Nephrol. in $2005^{40}$ described that the different levels of podocyte depletion assess the level of glomerulosclerosis in the transgenic rat model. This means that inducible, partially sensitive, and frequently relapsing NS are all on a spectrum with steroid-resistant NS. Furthermore, SSNS maybe a multifactorial disease and not a simple Mendelian disorder. It may also be influenced by multiple modifying genes and environmental factors such as infections and nature of host immune responses, besides mutation in a particular gene. Therefore, the nephrotic phenotype of Itsn $2-L$ knockout mice may vary greatly depending on genetic background of mouse strain and environmental factors, configuring a phenotypic spectrum of the Itsn2related NS. Therefore, it is not surprising that Itsn2-L knockout mouse did not mimic the symptoms of the patients with ITSN2 mutations.

In summary, we detected recessive mutations in cohort of individuals with partially treatment sensitive NS, which places this pathogenesis at the interphase between SSNS and SRNS. Definition of this pathogenic pathway opens inroads into defining therapeutic targets for SRNS, for which currently no efficient treatment exists.

\section{Methods}

Study participants. We obtained blood samples and pedigrees following informed consent from individuals with NS. Approval for human subjects' research was obtained from Institutional Review Boards of the University of Michigan, the Boston Children's Hospital and other local IRBs. The diagnosis of NS was based on published clinical criteria. This study was also approved by the Ethical Committee of Tohoku University Graduate School of Medicine. Written informed consent was obtained from all members of the Japanese families and the individuals with SSNS for the use of their clinical data and blood samples.

Linkage analysis. For genome-wide homozygosity mapping the GeneChip ${ }^{\circledR}$ Human Mapping $250 \mathrm{k}$ StyI Array from Affymetrix was used. Non-parametric LOD scores were calculated using a modified version of the program GENEHUNTER 2.1 $1^{41,42}$ through stepwise use of a sliding window with sets of 110 SNPs and the program ALLEGRO ${ }^{43}$ in order to identify regions of homozygosity as described $^{10,44}$ using a disease allele frequency of 0.0001 and Caucasian marker allele frequencies.

Whole exome sequencing. Whole exome sequencing (WES) and variant burden analysis was performed as described ${ }^{45}$. In brief, genomic DNA was isolated from blood lymphocytes and subjected to exome capture using Agilent SureSelect ${ }^{\text {Tix }}$ human exome capture arrays (Life technologies ${ }^{\mathrm{TM}}$ ) followed by next generation sequencing on the Illumina ${ }^{\mathrm{TM}}$ sequencing platform. For family from Japan, exon capture was performed with SureSelectXT Human All Exon V4 $(51 \mathrm{Mb}) \mathrm{Kits}$ (Agilent Technologies). Exome libraries were sequenced on a HiSeq2000 (Illumina) according to the manufacturer's instructions. Paired reads were aligned to the hg19 human reference using Novoalign V2.08.05 (http://novocraft.com), and single nucleotide variants (SNVs) and insertions and/or deletions (indels) were called using the Genome Analysis Toolkit (GATK) v1.6-13 ${ }^{46}$.

Fig. 4 ITSN1 and ITSN2 are GEFs for Cdc42, regulating NS-related podocyte function, and Itsn2-/- mice develop incompletely penetrant NS upon LPS injection. a Myc-tagged ITSN1-WT increased active Cdc42 levels upon overexpression in HEK293T cells as compared to mock, whereas three out of five ITSN1 mutants from pTSNS patients failed to show any significant increase in active Cdc42 upon overexpression. b Active Cdc42 level was measured in COS7 cells. Myc-tagged ITSN2-WT overexpression increased active Cdc42 levels as compared to Mock. However, all three ITSN2 mutants from pTSNS patients failed to show any significant increase in active Cdc42 upon overexpression. Error bars are defined as the standard error of at least four independent experiments. One-way ANOVA with Dunnet's post hoc test vs. wild-type (WT) ITSN2 expressing control. c Filopodia induction in cultured human podocytes was quantified by counting the transfected cells with filopodia and showed as a percentage of cells. For each construct, 50 transfected cells from three independent experiments were analyzed. One-way ANOVA with Dunnet's post hoc test vs. WT ITSN1 expressing control. Data are presented as the mean \pm SEM. ${ }^{\star} P<0.05,{ }^{\star \star} P<0.01,{ }^{\star \star \star} P<0.001,{ }^{\star \star \star \star} P P<0.0001$. d Filopodia induction in cultured human podocytes was quantified by counting the transfected cells with filopodia and showed as a percentage of cells. For each construct, 50 transfected cells from three independent experiments were analyzed. One-way ANOVA with Dunnet's post hoc test vs. WT ITSN2 expressing control. Data are presented as the mean \pm SEM. * $P<$ $0.05,{ }^{\star \star} P<0.01,{ }^{\star \star \star} P<0.001,{ }^{\star \star \star \star} P<0.0001$. e Kidneys of WT and Itsn $2^{L-/ L-}$ mice were stained with periodic acid Schiff. The glomeruli of Itsn $2^{L-/ L-}$ mice showed normal findings, similar to WT. f Urine albumin of Itsn $2^{L-/ L-}$, Itsn $2^{+/ L-}$, and WT mice was measured before and after LPS injection. Albuminuria level was increased in Itsn $2^{L-} / L-$ mice from 12 to $48 \mathrm{~h}$ after LPS injection compared with WT and Itsn2 $+/ L-$ mice. Two-tailed Student's $t$ tests. Data are presented as the mean \pm SEM. ${ }^{\star} P<0.05 . \mathbf{g}$ Representative electron microscopy images. LPS injection-induced foot process (FP) effacement in both WT and Itsn $2^{L-/ L-}$ mice within $24 \mathrm{~h}$. At $48 \mathrm{~h}$ after LPS injection, FP effacement was still observed in Itsn $2-/ L-$ mice, although WT mice recovered from FP effacement 
Targeted sequencing. DNA was obtained from 153 individuals with SSNS by the group from Japan. The 153 patients screened for ITSN2 mutations were screened by panel sequencing rather than by whole exome sequencing. We designed a custom panel of 21 genes (total target bases: $183.44 \mathrm{kbp}$, Supplementary Table 7) using the SureDesign Tool (Agilent Technologies). Target capture was performed with the HaloPlex target enrichment system (Agilent Technologies). Libraries were sequenced on a MiSeq or HiSeq2000 (Illumina). Mapping and variant call were performed using SureCall v2.1.0.21. Variants in ITSN2 were manually verified using an Integrative Genomics Viewer (IGV), and nonsynonymous mutations were retained and filtered for a MAF $<0.01$ in the 1000 Genomes database and HGVD.

Mutation calling. Sequence reads were mapped against the human reference genome (NCBI build 37/hg19) using CLC Genomics Workbench (version 6.5.1) software (CLC bio). Variants with minor allele frequencies $<1 \%$ in the dbSNP (Version 137) database were selected and annotated for impact on the encoded protein and for conservation of the reference base and amino acid among orthologs across phylogeny. Mutation calling was performed by geneticists/cell biologists, who had knowledge of the clinical phenotypes and pedigree structure, as well as experience with homozygosity mapping and exome evaluation.

\section{High-throughput mutation analysis by array-based multiplex PCR and NGS} We used PCR-based 48.48 Access Array microfluidic technology (Fluidigm ${ }^{\mathrm{TM}}$ ) with consecutive next generation sequencing. We applied a 12 -fold primer multiplexing approach allowing PCR-based amplification for 48 DNA samples simultaneously in 576 amplicons ${ }^{12,13}$. A total of 2000 individuals with NS were analyzed, among them subset of 800 individuals with SSNS. After amplification of all targeted coding and splice site regions, sample-derived products were indexed with 384 different 10 bp-barcodes in a subsequent PCR. Finally, $2 \times 250$ bp pairedend sequencing was performed on an Illumina ${ }^{\mathrm{TM}} \mathrm{MiSeq}$ instrument. Bioinformatic analysis was conducted using CLC-Genomics-Workbench ${ }^{\mathrm{TM}}$ software. Potential mutations were confirmed by Sanger sequencing and evaluated for segregation.

cDNA cloning. Rat Magi2 (S-SCAM) full-length cDNA was gifted by Dr. Min Loo, Yonsei University College of Medicine, Korea. Human TNS2 isoform 2 full-length cDNA was subcloned by PCR from human full-length cDNA (cDNA clone MGC: 165058, IMAGE: 40148847). Mouse TNS2 was subcloned from mouse TNS2 fulllength ORF cDNA (Cat No: MR211954, Origene). Human DLC1 subcloned from human fetal brain cDNA library (Clontech). Human CDK20 full-length was subcloned from human full-length cDNA (cDNA clone MGC: 3757, IMAGE: 3605918). Mouse Cdk20 was subcloned from mouse full-length Cdk20 (cDNA clone, MGC: 38901, IMAGE: 5362198). Human ITSN1 full-length was subcloned from human full-length cDNA (cDNA clone MGC: 134949 IMAGE: 40073781). Expression vectors were produced using LR clonase (Invitrogen ${ }^{\circledR}$ ) following the manufacturer's instruction. The coding sequences of human ITSN2- $L$ was purchased from Origene, and the ITSN2- $L$ with the N-terminal FLAG tag (FLAGITSN2-L) or FLAG (mock) was subcloned into pCAGEN using the In-Fusion ${ }^{\circledR}$ HD Cloning Kit (TaKaRa, Clontech). The following expression vectors were used in this publication: pRK5-N-Myc, pCDNA6.2-N-GFP, pCMV6-AN-DDK (Origene) or pCAGEN. pCAGEN was a gift from Connie Cepko (Addgene plasmid \# 11160) 47 . Clones reflecting the mutations identified in individuals with NS were introduced in the cDNA constructed by using the Quick change II XL site-directed mutagenesis kit, Agilent Technologies or by the PrimeSTAR ${ }^{\circledR}$ Mutagenesis Basal Kit (TaKaRa). Final sequences were confirmed by sequencing. pRK5-Myc-Cdc42WT was a gift from Gary Bokoch (Addgene plasmid \# 12972).

Cell lines. Experiments shown in this publication were performed in HEK293T cells, and immortalized human podocytes. HEK293T cells were purchased from the ATCC biological resource center. Human immortalized podocytes were a kind gift from Moin Saleem, University of Bristol, Bristol, UK, and were cultured as previously described ${ }^{48}$. Cell lines were tested for mycoplasma contamination on a quarterly basis.

Coimmunoprecipitation. Coimmunoprecipitation experiments were performed as described previously ${ }^{49}$. Briefly, cell lysates were pre-cleared with protein G or A beads. Then, cell lysates were mixed with the appropriate antibodies and incubated overnight at $4{ }^{\circ} \mathrm{C}$ in lysis buffer containing the complete protease inhibitor mixture. Immune complexes were collected by binding to mixed protein $\mathrm{G}$ or A beads and washed four times with lysis buffer prior to immunoblotting. Coimmunoprecipitation of GFP fusion proteins was performed using Chromotek-GFP-Trap ${ }^{\circledast}$ Agarose Beads, allele bioscience.

Knockdown in HEK293T cells and human podocytes. Knockdown efficiencies for respective siRNAs in HEK293T cells for TNS2, DLC1, CDK20, and ITSN1 are shown in Supplementary Fig. 25 and for CAV1 in Supplementary Fig. 26. Efficiency of transient knockdown of MAGI2, TNS2, DLC1, CDK20, and ITSN1 in undifferentiated human podocytes was confirmed by measuring mRNA levels by realtime PCR using TaqMan probes (Supplementary Fig. 27). All target sequences are listed in Supplementary Table 8. Knockdown experiments were performed $48 \mathrm{~h}$ after transfection. shRNA targeting human TNS2 and DLC1 were subcloned into pSirenRetroQ for retroviral transduction using HEK293T cells. Forty eight hour after transduction, puromycin was added to the medium at a final concentration of $4 \mu \mathrm{g} / \mathrm{ml}$ for selection of transduced cells. See Supplementary Table 8 for target sequences and Supplementary Fig. 28 for knockdown efficiency.

Cell migration assay. Briefly, the migration assays were performed using IncuCyte $^{\mathrm{ma}}$ video-microscopy system (Essen Biosciences) according to the manufacturer's instructions. IMCD3 cells or human podocytes were transfected with siRNAs for DLC1, MAGI2, CDK20, or TNS2. For rescue experiments (Fig. 2f), knockdown of Dlc1 in IMCD3 cells was followed by transfection with mock-myc or human DLC1 WT or mutants. Results were plotted as percentage of wound confluence (relative podocyte migration) vs. time. Each experiment was performed at least in triplicates, and repeated two times independently. Results are presented as mean with standard deviation.

G-LISA Rho, Rac, and Cdc42 activation assays. Cells were transfected in six-well plates with WT constructs or mock using Lipofectamine-2000 for overexpression experiments, or with siRNA against the target or scrambled control using Lipofectamine-RNAiMAX for knockdown experiments. Transfected cells were incubated in DMEM (HEK293T) or RPMI (human podocytes) with 10\% fetal bovine serum for $8 \mathrm{~h}$ and then in serum-free medium for $24 \mathrm{~h}$. Rho, Rac, or Cdc42 activity was determined using a colorimetric G-LISA Rho or Rac or Cdc42 Activation Assay Biochem kit (Cytoskeleton), according to the manufacturer's instructions.

Measurement of filopodia induction in human podocytes. Human podocyte cells on glass coverslips were transfected with CDC42 constructs and ITSN1 or ITSN2 constructs or pCAGEN-FLAG-mock using Lipofectamine $2000^{\circledR}$ in six-well plates and were incubated in RPMI with $10 \%$ fetal bovine serum for $8 \mathrm{~h}$ and then in serum-free medium for $24 \mathrm{~h}$. Cells were fixed in $4 \%$ paraformaldehyde (PFA) for $10 \mathrm{~min}$ and containing $0.25 \%$ Triton X-100. Cells were stained for $60 \mathrm{~min}$ at room temperature (RT) with mouse monoclonal anti-FLAG antibodies (1:500, SigmaAldrich, F3165) and rabbit polyclonal anti-Myc antibodies (1:500, santa cruz, sc789), washed with phosphate-buffered saline (PBS) and incubated for $1 \mathrm{~h}$ at RT with Alexa Fluor 647 anti-mouse secondary antibody (1:500, life technologies, A31571), Alexa Fluor 594 anti-rabbit secondary antibody (1:500, life technologies, A21207) and Alexa Fluor 488 Phalloidin (1:40, Invitrogen, A12379). Coverslips were then mounted on slides with ProLong Gold antifade reagent with DAPI (Invitrogen). Confocal imaging was performed using Leica SP5X system with an upright DM6000 microscope and images were processed with the Leica AF software suite. Filopodia were defined as thin, finger-like protrusive structures ${ }^{32}$. The ratio of cells with filopodia was calculated.

Circular dichroism (CD). The WT-DLC1 SAM domain (amino acid number $17-76$ ) was cloned in pETM vector as described ${ }^{50}$. A19V point mutation was incorporated in WT construct by site directed mutagenesis. The mutated DNA was transformed into Escherichia coli XL1-blue cells and plated on antibiotic containing agar plate. The correct in frame mutations were confirmed by sequencing of selected clones. The pET-M plasmids were transformed into E. coli BL21 (DE3) cells and the cells were grown in LB broth at $37^{\circ} \mathrm{C}$. Protein expression was induced by adding $1 \mathrm{mM}$ IPTG. The cells were harvested and lysed by sonication in lysis buffer containing $20 \mathrm{mM}$ Tris- $\mathrm{HCl}$ (pH 8), $200 \mathrm{mM} \mathrm{NaCl}, 5 \mathrm{mM}$ DTT, $1 \%$ Triton $\mathrm{X}-100,5 \%$ Glycerol, and protease inhibitor (Roche). The cleared lysates was transferred to previously washed Ni-NTA beads for overnight binding. Protein with $6 \mathrm{x}$ His tag was eluted from beads in two step elution in buffer $20 \mathrm{mM}$ Tris$\mathrm{HCl}(\mathrm{pH} 8), 150 \mathrm{mM} \mathrm{NaCl}, 5 \%$ Glycerol, $5 \mathrm{mM}$ DTT, and 200-300 $\mu \mathrm{M}$ Immidazole. The eluted protein was loaded on pre-equilibrated Superdex 75 column for further purification by size exclusion chromatography. $\mathrm{CD}$ spectrum was collected for WT and mutant protein samples on a Jasco J-810 CD spectropolarimeter with protein concentration $\sim 30 \mu \mathrm{M}$ in $10 \mathrm{mM}$ Tris ( $\mathrm{pH} 8$ ) and $75 \mathrm{mM} \mathrm{NaCl}$. Spectra were recorded in a cuvette of path length $0.1 \mathrm{~mm}$. The melting curves were obtained by recording the $\mathrm{CD}$ signals at $222 \mathrm{~nm}$ across the temperature range from 20 to $90^{\circ} \mathrm{C}$. The reading was taken at every $2^{\circ} \mathrm{C}$ increase of temperature.

Antibodies. For immunofluorescence experiments the following primary antibodies were used: rabbit anti-TNS2 (Sigma, HPA034659, 1:100); mouse anti-DLC1 (BD Biosciences, clone 3, 1:200); rabbit anti-ITSN1 (Abcam, ab118262, 1;100); and rabbit anti-ITSN2 (Abcam, ab176592, 1:100). Donkey anti-goat Alexa 488 (1:500) and Alexa-594 (1:500) conjugated secondary antibodies, and DAPI (4',6-Diamidino-2-Phenylindole, Dihydrochloride, 1:10,000) were obtained from Invitrogen (Supplementary Fig. 29). For immunoblotting the following primary antibodies were used: CDK20 rabbit polyclonal (Novus, NBP1-91214, 1;1000); ITSN1, rabbit polyclonal (Abcam, ab118262, 1:1000). Antibodies against TNS2 or DLC1 used here have been described ${ }^{51,52}$ (Supplementary Fig. 25). HRP labeled secondary antibodies were purchased from Santa Cruz. 
Immunofluorescence and confocal microscopy in cell lines. For immunostaining human immortalized podocytes were seeded on coverglasses, and grown at permissive temperature. For overexpression studies human podocytes were transiently transfected using lipofectamine $2000^{\circledR}$ following the manufacturer's instructions. Experiments were performed $24-48 \mathrm{~h}$ after transfection. Cells were fixed and permeabilized for $10 \mathrm{~min}$ using $4 \%$ paraformaldehyde and $0.25 \%$ Triton$\mathrm{X} 100$. After blocking, cells were incubated over-night at $4{ }^{\circ} \mathrm{C}$. The cells were incubated in secondary antibodies for $90 \mathrm{~min}$ at room-temperature, followed by 5 min staining with $1 \times$ DAPI/PBS. Confocal imaging was performed using Leica SP5X system with an upright DM6000 microscope and images were processed with the Leica AF software suite.

Itsn2-L knockout mice. All animal experiment protocols were approved by The Institutional Animal Care and Use Committee of Tohoku University. Mice were housed in a temperature- and humidity-controlled room, with a 12-h light/dark cycle and were allowed access to food and water ad libitum. Generation of the Itsn2-L knockout mice were entrusted to Unitech (Chiba, Japan). The targeting vector replaced a region that included exon 31 of Itsn2 (NC_000078; Supplementary Fig. 24) so that the ITSN2 long isoform, which has Cdc42-GEF activity, was absent throughout the entire body of the mice. The knockout mice were produced on a background of C57BL/6. Genotyping was performed by real-time PCR using a HybProbe assay as previously described ${ }^{53}$. The primers and probes were designed as follows: 5'-CTTCCTCACTGAAGCAGAC-3' (forward primer for wild-type), 5'-TGCTCCAGACTGCCTTG-3' (forward primer for knockout), 5'TGCTCCTGCTTGGAGAT-3' (reverse primer), 5'-CGGGGGCTGCTCAGCAAFITC-3' (upstream probe for wild-type), 5'-TCAAGCTTATCGATGATATCAFITC-3' (upstream probe for knockout), 5'-LCRed640-ACAACTTC-

CAACTCTGTTCTGTC-P-3' (downstream probe). Template DNA was extracted from tails. To confirm the loss of Itsn2-L mRNA transcripts, RT-PCR was performed. Total RNA was isolated from the kidney, liver, heart, and brain of Itsn2knockout mice and wild type mice using an RNeasy Mini Kit (Qiagen). Using a PrimeScript II High Fidelity RT-PCR Kit (TaKaRa Bio), 500 ng of RNA was reverse transcribed. Total RNA was amplified using the following primer set: $5^{\prime}$ CACGCTGTATGTCAAGTGATTGC-3' (forward primer) 5'-

CATTGGGTCACTGTTGACTTGG-3' (reverse primer for Itsn2 short) 5' CTTCAGTGAGGAAGCCTGACTCAG-3' (reverse primer for Itsn2 long).

LPS-induced proteinuria and electron microscopy. Transient proteinuria was induced by LPS injection as described ${ }^{54}$. We injected 4- to 6-week-old female C57BL/6 $(n=18)$, Itsn $2^{+/ L-}(n=10)$, and Itsn $2^{\mathrm{L}-/ \mathrm{L}-}(n=10)$ mice with LPS $(200 \mu \mathrm{g}$ intraperitoneally (i.p.); SIGMA Aldrich). Urine samples were obtained from individual mice at various time points before $(0 \mathrm{~h})$ and after $(12,24,36,48,60$, and 72 h) the injection of LPS. Urinary albumin and creatinine were measured using an Albuwell M kit and the creatinine companion kit (Exocell) according to the manufacturer's protocol. Albuminuria was calculated as micrograms of albumin per milligram creatinine. Kidneys were dissected from WT and Itsn2- $\mathrm{L}^{-/-}$mice 24 and $48 \mathrm{~h}$ after LPS injection. Specimens for EM were fixed with 2\% PFA and 2.5\% glutaraldehyde, washed with $0.1 \mathrm{M}$ cacodylate buffer, and incubated with $1 \% \mathrm{OsO}_{4}$ for $90 \mathrm{~min}$. The sections were counterstained with $1 \%$ uranyl acetate for $50 \mathrm{~min}$ dehydrated in a graded series of ethanol $(50,60,70,80,90$, and $95 \%)$, and finally embedded in Epon 812. Ultrathin sections $(80 \mathrm{~nm})$ were cut with a Leica EM UC7 (Leica) and mounted in copper grids. Images were obtained using a transmission electron microscope (H-7600; Hitachi High-Technologies). The mice experiments were not blinded.

Statistical analysis. Statistical analysis was performed with Graph Pad Prism. One-way ANOVA with Dunnet's post hoc test was performed. Displayed in the figure are the mean values of all technical replicates for each of the independent experiments. Error bars represent s.d. $P$ values $<0.05$ were considered statistically significant.

Bioinformatics. Genetic location is according to the February 2009 Human Genome Browser data (http://www.genome.ucsc.edu).

Data availability. All data generated or analyzed during this study are available in this published article (and its Supplementary Information Files) or from the corresponding author upon reasonable request.

Received: 19 May 2017 Accepted: 7 April 2018

Published online: 17 May 2018

\section{References}

1. Smith, J. M., Stablein, D. M., Munoz, R., Hebert, D. \& McDonald, R. A. Contributions of the transplant registry: the 2006 annual report of the North
American pediatric renal trials and collaborative studies (NAPRTCS). Pediatr. Transplant. 11, 366-373 (2007).

2. Lovric, S., Ashraf, S., Tan, W. \& Hildebrandt, F. Genetic testing in steroidresistant nephrotic syndrome: when and how? Nephrol. Dial. Transplant. 31, 1802-1813 (2015).

3. Sadowski, C. E. et al. A single-gene cause in $29.5 \%$ of cases of steroid-resistant nephrotic syndrome. J. Am. Soc. Nephrol. 26, 1279-1289 (2015).

4. Gee, H. Y. et al. ARHGDIA mutations cause nephrotic syndrome via defective RHO GTPase signaling. J. Clin. Invest. 123, 3243-3253 (2013).

5. Gee, H. Y. et al. KANK deficiency leads to podocyte dysfunction and nephrotic syndrome. J. Clin. Invest. 125, 2375-2384 (2015).

6. Ashraf, S. et al. ADCK4 mutations promote steroid-resistant nephrotic syndrome through CoQ10 biosynthesis disruption. J. Clin. Invest. 123, 5179-5189 (2013).

7. Lovric, S. et al. Mutations in sphingosine-1-phosphate lyase cause nephrosis with ichthyosis and adrenal insufficiency. J. Clin. Invest. 127, 912-928 (2017).

8. Gee, H. Y. et al. Mutations in EMP2 cause childhood-onset nephrotic syndrome. Am. J. Hum. Genet. 94, 884-890 (2014).

9. Sadowski, C. E. et al. A single-gene cause in $29.5 \%$ of cases of steroid-resistant nephrotic syndrome. J. Am. Soc. Nephrol. 26, 1279-1289 (2014).

10. Hildebrandt, F. et al. A systematic approach to mapping recessive disease genes in individuals from outbred populations. PLOS Genet. 5, e1000353 (2009).

11. Otto, E. A. et al. Candidate exome capture identifies mutation of SDCCAG8 as the cause of a retinal-renal ciliopathy. Nat. Genet. 42, 840-850 (2010).

12. Halbritter, J. et al. High-throughput mutation analysis in patients with a nephronophthisis-associated ciliopathy applying multiplexed barcoded arraybased PCR amplification and next-generation sequencing. J. Med. Genet. 49, 756-767 (2012).

13. Halbritter, J. et al. Identification of 99 novel mutations in a worldwide cohort of 1,056 patients with a nephronophthisis-related ciliopathy. Hum. Genet. 132, 865-884 (2013).

14. Balbas, M. D. et al. MAGI-2 scaffold protein is critical for kidney barrier function. Proc. Natl Acad. Sci. USA 111, 14876-14881 (2014).

15. Ihara, K. et al. MAGI-2 is critical for the formation and maintenance of the glomerular filtration barrier in mouse kidney. Am. J. Pathol. 184, 2699-2708 (2014).

16. Lefebvre, J. et al. Alternatively spliced isoforms of WT1 control podocytespecific gene expression. Kidney Int. 88, 321-331 (2015).

17. Bierzynska, A. et al. MAGI2 mutations cause congenital nephrotic syndrome. J. Am. Soc. Nephrol. 28, 1614-1621 (2016).

18. Yam, J. W., Ko, F. C., Chan, C. Y., Jin, D. Y. \& Ng, I. O. Interaction of deleted in liver cancer 1 with tensin2 in caveolae and implications in tumor suppression. Cancer Res. 66, 8367-8372 (2006).

19. Chan, L. K., Ko, F. C., Ng, I. O. \& Yam, J. W. Deleted in liver cancer 1 (DLC1) utilizes a novel binding site for Tensin2 PTB domain interaction and is required for tumor-suppressive function. PLOS One 4, e5572 (2009).

20. Yu, Y. et al. Mice deficient for the chromosome 21 ortholog Itsn1 exhibit vesicle-trafficking abnormalities. Hum. Mol. Genet. 17, 3281-3290 (2008).

21. Wu, X. et al. Evidence for regulation of the PTEN tumor suppressor by a membrane-localized multi-PDZ domain containing scaffold protein MAGI-2. Proc. Natl Acad. Sci. USA 97, 4233-4238 (2000).

22. Tripathi, B. K. et al. CDK5 is a major regulator of the tumor suppressor DLC1 J. Cell. Biol. 207, 627-642 (2014).

23. $\mathrm{Du}, \mathrm{X}$. et al. Functional interaction of tumor suppressor DLC1 and caveolin-1 in cancer cells. Cancer Res. 72, 4405-4416 (2012).

24. Forbes, A. et al. The tetraspan protein EMP2 regulates expression of caveolin1. J. Biol. Chem. 282, 26542-26551 (2007).

25. Wan, X. et al. Loss of epithelial membrane protein 2 aggravates podocyte injury via upregulation of caveolin-1. J. Am. Soc. Nephrol. 27, 1066-1075 (2016).

26. Hood, J. D. \& Cheresh, D. A. Role of integrins in cell invasion and migration. Nat. Rev. Cancer 2, 91-100 (2002).

27. Perico, L., Conti, S., Benigni, A. \& Remuzzi, G. Podocyte-actin dynamics in health and disease. Nat. Rev. Nephrol. 12, 692-710 (2016).

28. Faul, C., Asanuma, K., Yanagida-Asanuma, E., Kim, K. \& Mundel, P. Actin up: regulation of podocyte structure and function by components of the actin cytoskeleton. Trends Cell. Biol. 17, 428-437 (2007).

29. Leettola, C. N., Knight, M. J., Cascio, D., Hoffman, S. \& Bowie, J. U. Characterization of the SAM domain of the PKD-related protein ANKS6 and its interaction with ANKS3. BMC Struct. Biol. 14, 17 (2014).

30. Gee, H. Y. et al. FAT1 mutations cause a glomerulotubular nephropathy. Nat. Commun. 7, 10822 (2016)

31. McGavin, M. K. et al. The intersectin 2 adaptor links Wiskott Aldrich Syndrome protein (WASp)-mediated actin polymerization to T cell antigen receptor endocytosis. J. Exp. Med. 194, 1777-1787 (2001).

32. Mattila, P. K. \& Lappalainen, P. Filopodia: molecular architecture and cellular functions. Nat. Rev. Mol. Cell. Biol. 9, 446-454 (2008). 
33. Krugmann, S. et al. Cdc42 induces filopodia by promoting the formation of an IRSp53:Mena complex. Curr. Biol. 11, 1645-1655 (2001).

34. Alwadhi, R. K., Mathew, J. L. \& Rath, B. Clinical profile of children with nephrotic syndrome not on glucorticoid therapy, but presenting with infection. J. Paediatr. Child. Health 40, 28-32 (2004).

35. Reiser, J. et al. Induction of B7-1 in podocytes is associated with nephrotic syndrome. J. Clin. Invest. 113, 1390-1397 (2004).

36. Wang, L. et al. Mechanisms of the proteinuria induced by Rho GTPases. Kidney Int. 81, 1075-1085 (2012).

37. Scott, R. P. et al. Podocyte-specific loss of Cdc42 leads to congenital nephropathy. J. Am. Soc. Nephrol. 23, 1149-1154 (2012).

38. Garcia-Mata, R., Boulter, E. \& Burridge, K. The 'invisible hand': regulation of RHO GTPases by RHOGDIs. Nat. Rev. Mol. Cell. Biol. 12, 493-504 (2011).

39. Nishino, T. et al. The 129 genetic background affects susceptibility to glomerulosclerosis in tensin2-deficient mice. Biomed. Res. 33, 53-56 (2012).

40. Wharram, B. L. et al. Podocyte depletion causes glomerulosclerosis: diphtheria toxin-induced podocyte depletion in rats expressing human diphtheria toxin receptor transgene. J. Am. Soc. Nephrol. 16, 2941-2952 (2005).

41. Strauch, K. et al. Parametric and nonparametric multipoint linkage analysis with imprinting and two-locus-trait models: application to mite sensitization. Am. J. Hum. Genet. 66, 1945-1957 (2000).

42. Kruglyak, L., Daly, M. J., Reeve-Daly, M. P. \& Lander, E. S. Parametric and nonparametric linkage analysis: a unified multipoint approach. Am. J. Hum. Genet. 58, 1347-1363 (1996).

43. Gudbjartsson, D. F., Jonasson, K., Frigge, M. L. \& Kong, A. Allegro, a new computer program for multipoint linkage analysis. Nat. Genet. 25, 12-13 (2000).

44. Sayer, J. A. et al. The centrosomal protein nephrocystin-6 is mutated in Joubert syndrome and activates transcription factor ATF4. Nat. Genet. 38, 674-681 (2006).

45. Boyden, L. M. et al. Mutations in kelch-like 3 and cullin 3 cause hypertension and electrolyte abnormalities. Nature 482, 98-102 (2012).

46. McKenna, A. et al. The Genome Analysis Toolkit: a MapReduce framework for analyzing next-generation DNA sequencing data. Genome Res. 20, 1297-1303 (2010).

47. Matsuda, T. \& Cepko, C. L. Electroporation and RNA interference in the rodent retina in vivo and in vitro. Proc. Natl Acad. Sci. USA 101, 16-22 (2004).

48. Saleem, M. A. et al. A conditionally immortalized human podocyte cell line demonstrating nephrin and podocin expression. J. Am. Soc. Nephrol. 13, 630-638 (2002).

49. Zariwala, M. A. et al. ZMYND10 is mutated in primary ciliary dyskinesia and interacts with LRRC6. Am. J. Hum. Genet. 93, 336-345 (2013).

50. Zhong, D. et al. The SAM domain of the RhoGAP DLC1 binds EF1A1 to regulate cell migration. J. Cell. Sci. 122, 414-424 (2009).

51. Hafizi, S., Ibraimi, F. \& Dahlback, B. C1-TEN is a negative regulator of the Akt/PKB signal transduction pathway and inhibits cell survival, proliferation, and migration. Faseb. J. 19, 971-973 (2005).

52. Li, G. et al. Full activity of the deleted in liver cancer 1 (DLC1) tumor suppressor depends on an LD-like motif that binds talin and focal adhesion kinase (FAK). Proc. Natl Acad. Sci. USA 108, 17129-17134 (2011).

53. Kikuchi, A. et al. Simple and rapid genetic testing for citrin deficiency by screening 11 prevalent mutations in SLC25A13. Mol. Genet. Metab. 105, 553-558 (2012).

54. Asanuma, K. et al. Synaptopodin regulates the actin-bundling activity of alpha-actinin in an isoform-specific manner. J. Clin. Invest. 115, 1188-1198 (2005).

\section{Acknowledgements}

We are grateful to study individuals for their contribution. We acknowledge the Yale Center for Mendelian Genomics for whole exome sequencing. This research was supported by grants from the National Institutes of Health to F.H. (DK076683) and by the Howard Hughes Medical Institute to F.H. F.H. is the William E. Harmon Professor of Pediatrics. E.W. is supported by the German National Academy of Sciences Leopoldina (LPDS-2015-07). W.T. is supported by the ASN Foundation for Kidney Research. T.H. is supported by the DFG-fellowship (HE 7456/1-1). T.J.S. is supported by the Deutsche
Forschungsgemeinschaft (Jo 1324/1-1). H.Y.G. is supported by the National Research Foundation of Korea, Ministry of Science, ICT, and Future planning (2015R1D1A1A01056685) and faculty seed money from the Yonsei University College of Medicine (2015-32-0047). A.T.v.d.V. is supported by the DFG-fellowship (VE 196/1-1). We thank Yoko Chiba, Kumi Ito, Miyuki Tsuda, Mami Kikuchi, Makiko Nakagawa, Yoko Tateda and Kiyotaka Kuroda for their technical assistance. This work was supported by grants (17ek0109151h0003 and 17ek0109278h0001) from Japan Agency for Medical Research and Development (AMED) to S.K. We acknowledge the support of the Biomedical Research Unit of Tohoku University Hospital and the Biomedical Research Core and the Institute for Animal Experimentation of Tohoku University Graduate School of Medicine. N.A.S is supported by the Egyptian Group for Orphan Renal Diseases (EGORD). M.A.J. is supported by Department of Science and Technology, Govt. of India (DST-SERB). M.Z. was supported by the Deutsche Forschungsgemeinschaft (SFB423).

\section{Author contributions}

S.A., H.K., J.R., A.K., E.W., W.T., T.H., S.S., T.J.S., S.L., D.A.B., H.Y.G., D.S., A.M., C.E.S., W.L.P., A.D., A.T.v.d.V., J.M.S., S.M.M., R.P.L., S.K., and F.H. generated total genome linkage, performed exome capture and massively parallel sequencing, and performed whole exome evaluation and mutation analysis. S.A., J.R., J.A.L., B.K.T., J.W., K.C., L.K., D.R.L., and M.A.J. performed c-DNA cloning and/or co-immunoprecipitation experiments. S.A. and E.W. performed G-Lisa and drug treatment experiments. J.A.L., J.R., and J.K.W. performed immunofluorescence and subcellular localization studies in cell lines by confocal microscopy. S.A., E.W., W.T., and M.A. performed migration assays in immortalized human podocytes and IMCD3 cells. B.C.L. and A.B.G. performed circular dichroism experiments for DLC1. H.K., A.K., T.N., H.K., K.N., H.T., R.T., K.H., Y.K., T. T., R.F., K.N., Y.A., N.K., K.I., and S.K. recruited patients of Japanese origin, and performed whole exome sequencing and Itsn2 knockout mice studies. K.M., D.S., H.F., J.A. K., S.E.D., S.J., R.B., N.S., H.Z., A.R., S.R.W., T.J., J.S., A.S.K., A.B., N.A.S., M.A.J., Y.P., M. Z., and F.H. recruited patients and gathered detailed clinical information for the study. F. H. and S.A. conceived the study and wrote the manuscript, which was critically reviewed by all the authors.

\section{Additional information}

Supplementary Information accompanies this paper at https://doi.org/10.1038/s41467018-04193-w.

Competing interests: F.H. is a cofounder of Goldfinch-Bio. All remaining authors declare no competing interests.

Reprints and permission information is available online at http://npg.nature.com/ reprintsandpermissions/

Publisher's note: Springer Nature remains neutral with regard to jurisdictional claims in published maps and institutional affiliations.

\begin{abstract}
Open Access This article is licensed under a Creative Commons Attribution 4.0 International License, which permits use, sharing, adaptation, distribution and reproduction in any medium or format, as long as you give appropriate credit to the original author(s) and the source, provide a link to the Creative Commons license, and indicate if changes were made. The images or other third party material in this article are included in the article's Creative Commons license, unless indicated otherwise in a credit line to the material. If material is not included in the article's Creative Commons license and your intended use is not permitted by statutory regulation or exceeds the permitted use, you will need to obtain permission directly from the copyright holder. To view a copy of this license, visit http://creativecommons.org/ licenses/by/4.0/.
\end{abstract}

(C) The Author(s) 2018

Shazia Ashraf 1,2, Hiroki Kudo ${ }^{3}$, Jia Rao', Atsuo Kikuchi@ ${ }^{3}$, Eugen Widmeier', Jennifer A. Lawson', Weizhen Tan', Tobias Hermle, , Jillian K. Warejko', Shirlee Shril', Merlin Airik', Tilman Jobst-Schwann', Svjetlana Lovric', Daniela A. Braun', Heon Yung Gee 1,4, David Schapiro', Amar J. Majmundar', Carolin E. Sadowski', Werner L. Pabst', Ankana Daga ${ }^{1}$, Amelie T. van der Ven', Johanna M. Schmidt', Boon Chuan Low ${ }^{5,6}$, 
Anjali Bansal Gupta ${ }^{6}$, Brajendra K. Tripathi ${ }^{7}$, Jenny Wong ${ }^{8}$, Kirk Campbell ${ }^{8}$, Kay Metcalfe ${ }^{9}$, Denny Schanze (i) $^{10}$, Tetsuya Niihori ${ }^{11}$, Hiroshi Kaito ${ }^{12}$, Kandai Nozu ${ }^{12}$, Hiroyasu Tsukaguchi ${ }^{13}$, Ryojiro Tanaka (1) ${ }^{14}$, Kiyoshi Hamahira ${ }^{15}$, Yasuko Kobayashi ${ }^{16,17}$, Takumi Takizawa ${ }^{16}$, Ryo Funayama ${ }^{18}$, Keiko Nakayama ${ }^{18}$, Yoko Aoki ${ }^{11}$, Naonori Kumagai ${ }^{3}$, Kazumoto lijima ${ }^{12}$, Henry Fehrenbach ${ }^{19}$, Jameela A. Kari ${ }^{20}$, Sherif El Desoky ${ }^{20}$, Sawsan Jalalah ${ }^{21}$, Radovan Bogdanovic ${ }^{22}$, Nataša Stajić ${ }^{22}$, Hildegard Zappel ${ }^{23}$, Assel Rakhmetova ${ }^{24}$, Sharon-Rose Wassmer ${ }^{25}$, Therese Jungraithmayr ${ }^{26}$, Juergen Strehlau ${ }^{27}$, Aravind Selvin Kumar ${ }^{28}$, Arvind Bagga ${ }^{29}$, Neveen A. Soliman ${ }^{30}$, Shrikant M. Mane ${ }^{31}$, Lewis Kaufman ${ }^{8}$, Douglas R. Lowy7, Mohamad A. Jairajpuri ${ }^{7}$, Richard P. Lifton ${ }^{31,32}$, York Pei ${ }^{33}$, Martin Zenker ${ }^{10}$, Shigeo Kure ${ }^{3}$ \& Friedhelm Hildebrandt (iD ${ }^{1}$

${ }^{1}$ Department of Medicine, Boston Children's Hospital, Harvard Medical School, Boston, MA, USA. ²Department of Biosciences, Jamia Millia Islamia, New Delhi, India. ${ }^{3}$ Department of Pediatrics, Tohoku University School of Medicine, 1-1 Seiryo-machi, Aoba-ku, Sendai, Miyagi 980-8574, Japan. ${ }^{4}$ Department of Pharmacology, Brain Korea 21 PLUS Project for Medical Sciences, Yonsei University College of Medicine, Seoul 03722, Korea. ${ }^{5}$ Department of Biological Sciences, National University of Singapore, Singapore, Singapore. ${ }^{6}$ Mechanobiology Institute, National University of Singapore, Singapore, Singapore. ${ }^{2}$ Laboratory of Cellular Oncology, Center for Cancer Research, National Cancer Institute, National Institutes of Health, Bethesda, MD, USA. ${ }^{8}$ Division of Nephrology, Icahn School of Medicine at Mount Sinai, New York, NY, USA. ${ }^{9}$ Manchester Centre for Genomic Medicine, St Mary's Hospital, Central Manchester University Hospitals NHS Foundation Trust, Manchester Academic Health Science Centre, Manchester, UK. ${ }^{10}$ Institute of Human Genetics, University Hospital Magdeburg, Magdeburg, Germany. ${ }^{11}$ Department of Medical Genetics, Tohoku University School of Medicine, 1-1 Seiryo-machi, Aoba-ku, Sendai, Miyagi 980-8574, Japan. ${ }^{12}$ Department of Pediatrics, Kobe University Graduate School of Medicine, 7-5-2 Kusunoki-cho, Chuo-ku, Kobe 650-0017, Japan. ${ }^{13}$ 2nd Department of Internal Medicine, Kansai Medical University, 2-3-1 Shin-machi, Hirakata-shi, Osaka 573-1191, Japan. ${ }^{14}$ Department of Nephrology, Hyogo Prefectural Kobe Children's Hospital, 1-6-7 Minatojima-minamimachi, Chuo-ku, Kobe, Hyogo 650-0047, Japan. ${ }^{15}$ Department of Pediatrics, Himeji Red Cross Hospital, 1-12-1 Shimoteno, Himeji, Hyogo 670-8540, Japan. ${ }^{16}$ Department of Pediatrics, Gunma University Graduate School of Medicine, 3-39-22 Showa-machi, Maebashi, Gunma 371-8511, Japan. ${ }^{17}$ Academic Renal Unit, School of Clinical Science, University of Bristol, Dorothy Hodgkin Building, Whitson Street, Bristol BS1 3NY, United Kingdom. ${ }^{18}$ Division of Cell Proliferation, United Centers for Advanced Research and Translational Medicine, Tohoku University Graduate School of Medicine, Sendai, Miyagi 980-8575, Japan. ${ }^{19}$ Department of Pediatric Nephrology, Children's Hospital, Memmingen, Germany. ${ }^{20}$ Pediatric Nephrology Center of Excellence and Pediatric Department, King Abdulaziz University, Jeddah, Saudi Arabia. ${ }^{21}$ Pathology Department, King Abdulaziz University, Jeddah, Saudi Arabia. ${ }^{22}$ Institute for Mother and Child Health Care of Serbia "Dr Vukan Čupić", Department of Nephrology, University of Belgrade, Faculty of Medicine, Belgrade 11000, Serbia. ${ }^{23}$ Department for Paediatrics II, University of Göttingen, Göttingen, Germany. ${ }^{24}$ Department of Nephrology, Asfendiyarov Kazakh National Medical University, Almaty, Kazakhstan. ${ }^{25}$ Department of Nephrology, Luzerner Kantonsspital, Lucerne, Switzerland. ${ }^{26}$ Department of Pediatrics, University Medical Center Innsbruck, Innsbruck, Austria. ${ }^{27}$ Department of Pediatric Nephrology, Hannover Medical School, Hannover, Germany. ${ }^{28}$ Department of Pediatric Nephrology and Medical Genetics, Institute of Child Health and Hospital for Children, TN Dr.M.G.R. Medical University, Chennai, India. ${ }^{29}$ Division of Pediatric Nephrology, Department of Pediatrics, All India Institute of Medical Sciences, New Delhi, India. ${ }^{30}$ Department of Pediatrics, Center of Pediatric Nephrology \& Transplantation, Kasr Al Ainy School of Medicine, Cairo University, Cairo, Egypt. ${ }^{31}$ Department of Genetics, Yale University School of Medicine, New Haven, CT 06510, USA. ${ }^{32}$ Laboratory of Human Genetics and Genomics, The Rockefeller University, New York, NY 10065, USA. ${ }^{33}$ Division of Nephrology, University Health Network, and University of Toronto, Toronto, ON, Canada. These authors contributed equally: Shazia Ashraf, Hiroki Kudo. 